\title{
Devising an Innovative, Genotype-free Transformation Protocol for Recalcitrant Indica Rice Genotypes: Samba Mahsuri and Sundarbans' Salt- tolerant Indica Rice Cultivars White Getu and Hamilton to Establish an Ingenious Platform for CRISPR/Cas9-mediated Genome Editing
}

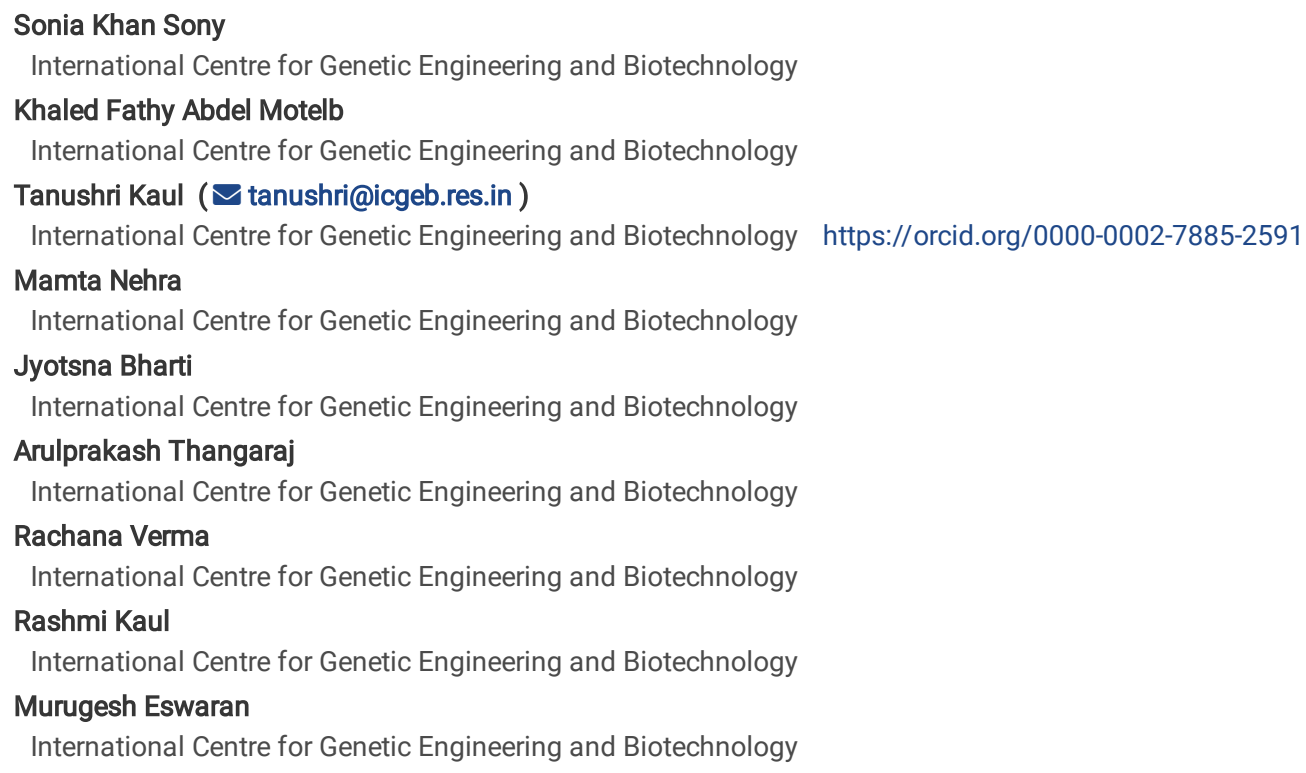

Research

Keywords: Rice, CRISPR, Cas9, Genome editing, Biotechnology, Regeneration, Transformation

Posted Date: November 29th, 2021

DOI: https://doi.org/10.21203/rs.3.rs-1078389/v1

License: (c) (1) This work is licensed under a Creative Commons Attribution 4.0 International License. Read Full License 


\section{Abstract}

Background

Plant genetic transformation involves in vitro callus induction \& regeneration strategies that are quintessential for introduction of novel agronomical traits employing CRISPR/Cas9-based genome editing. However, lack of effective regeneration and transformation techniques for indica rice cultivars pose as the foremost hurdle towards genetic improvement in rice crop. We devised an astounding road-map to genotype-independent and efficacious in vitro callus induction, transformation and shoot regeneration protocol that emerges as an optimal therapy, universally adaptable to invariably any rice cultivar, in order to establish an ingenious CRISPR/Cas9-based genome editing platform in this crop.

Results

We developed a genotype-independent regeneration and transformation protocol employing mature seed-derived calli for indica rice (one mega variety- Samba Mahsuri and two salt tolerant wild genotypes- White Getu \& Hamilton) genotypes to introduce important agronomical traits via CRISPR/Cas9-based genome editing system. MS- and N6-salt based media reinforced with 2,4-D (2.5 mg/L); dicamba (1.5 mg/L); TDZ (0.1 mg/L), proline (1000 mg/L), and glutamine (2.5 $\mathrm{mg} / \mathrm{L}$ ) exhibited highest percentage (95-98\%) of embryogenic calli initiation and development. Employing this novel protocol, we achieved unparalleled regeneration efficiencies within untransformed calli (90-94\%) and transformed calli (81-86\%) in these recalcitrant indica genotypes and significantly enhanced number of shoots (18-20) on MS medium containing BAP (1.5 mg/L), NAA (0.5 mg/L), TDZ (1.0 mg/L), zeatin ( $0.2 \mathrm{mg} / \mathrm{L})$ and proline (500 mg/L). We successfully transformed rice calli with pCAMBIA1300-based marker-free NICTK-1_pCRISPR-Cas 9 vector harbouring the cassette of plant codon optimized Cas 9 via biolistic approach that exhibited notably enhanced transformation efficiencies (67-69\%). The integration of Cas 9 gene into rice genome was validated by PCR, Southern blotting and Sanger sequencing analyses. The transgenic lines were phenotypically indistinguishable from the wild type as no significant differences in phenotypic performances were revealed between transgenic and wild type lines.

\section{Conclusion}

We devised a promising, time-efficient, universally adaptable, optimal hormonal-media therapy for triggering enhanced embryogenic callus formation, regeneration and transformation efficiencies, across recalcitrant indica rice genotypes.

\section{Background}

In recent times, food and nutritional security has become a major global challenge in underdeveloped and developing countries. Rice (Oryza sativa L.) is the second most popularly cultivated cereal grain after wheat, feeding more than $90 \%$ of the Asian population and half of the world's population consequently fulfilling more than $50-80 \%$ calorie requirement [1]. Indica and japonica are two major cultivated subspecies or eco-geographical races of rice [2], while indica subspecies are ubiquitously cultivated in the Southern and Southeastern countries in Asia, contributing to $80 \%$ of world's rice cultivation [3]. Even though, rice consumption has enormously risen at the rate of $1.8 \%$ per annum, the production rate has failed to meet the global demands, owing to umpteen factors for instance abiotic (52\%) and pest infestation (21-41\%) [4]. Hence, $70-100 \%$ escalation in the cereal food supply is pre-requisite to feed the predicted 9.8 billion of the world population by 2050 [1]. In order to enhance the yield potential of rice in the pre-genomic era, numerous attempts were undertaken to address this issue via conventional breeding technique. However, none of these efforts rendered the desired results due to the narrow genetic base, genetic instability, and non-availability of the resistance genes against biotic and abiotic stresses [5]. This prompted the researchers to focus on transgenic approaches, which can effectively address these problems as it permitted the transfer of genes across species barrier. Nevertheless, the inclusion of transgenes into a host plant genome is many a time unstable and nonspecific [6]. The past decade has ushered in an era of "Genome Sequencing and Genome Editing" with an upsurge of next-generation sequencing platforms and neoteric plant breeding approaches that has altered the manner in which breeding is performed via comprehending the genetics of the trait to expedite the genetic gains. Advent of the cutting edge CRISPR/Cas 9 genome editing technique has revolutionized the strategies for high precision genome editing of plants, specifically staple food and cash crops [7-12]. Thus, CRISPR/Cas9-based rice genome alterations have significantly fascinated crop scientists, globally [13-17]. A highly efficient and stable genetic transformation protocol is a pre-requisite for CRISPR/Cas9-based genome editing in a specific crop; wherein large-scale regeneration of plantlets through tissue culture is subjected to genetic modification. In umpteen crops, tissue culture-based genetic transformation has been commercially exploited for the development of transgenic varieties [18]. Although, certain rice genotypes exhibit high regeneration and transformation potentialities employing mature seeds; however, majority of the popular varieties pose challenges in achieving high regeneration and transformation percentages; for instance, genotype- and explant-dependency, physiological status of the explant, suitable composition of culture medium, and culture conditions [19]. Amongst all, the comprehensively used techniques for the integration of CRISPR-Cas9 reagents - sgRNA harbouring Cas9 vector and homology donor template into rice genome, direct DNA transfer employing biolistic approach via particle gun or shotgun bombardment $[12,19]$ and agro-mediated transformation $[20,21]$ are the most efficacious. The agro-mediated transformation approach is tedious \& labourintensive, and often regeneration of plantlets from transformed calli is the prime bottleneck. Furthermore, plants may reveal morphological abnormalities, somaclonal variations, genetic instability, and reduced fertility $[20,22]$. On the other hand, gene editing via biolistic method is sans tissue-specificity, effortless and effective for simultaneous transformation of more than one sgRNAs for desired modifications in the native plant genome via NHEJ or HDR pathways and RNP-complex mechanism. In addition, systematized biolistic transformation can maintain single or low copy (1-2) insertion in subsequent generations. Consequently, direct gene transfer employing biolistic approach permitted the development and assessment of elite rice transgenic cultivars, containing numerous agronomical importance genes, for instance, disease-, insect-, herbicide-resistance, and so on [23, 24].

In order to efficiently employ CRISPR/Cas9-based genome editing in rice, we have established an expeditious, robust, vigorously reproducible, potent plant transformation technique in three indica rice varieties i.e., Samba Mahsuri (SM)-a mega variety of Southern India and White Getu (WG) as well as Hamilton (HT) that are well-adapted to Eastern lowland saline areas utilizing mature seeds as explants. Subsequently, we developed transgenic rice lines harbouring the 
Cas9 gene and validated the lines by PCR and Southern blot analyses. Further, we monitored the comparative agronomic performances of the transgenic Cas9 and WT lines of the three crucial indica rice cultivars.

\section{Results And Discussion}

Stress either abiotic or biotic adversely affects rice productivity. Enhanced yield potential may be achieved by employing suitable biotechnological approaches. Plant transformation technique is indispensable for the introduction of novel traits in crops for nutritional and yield improvements thereby ensuring global food and nutritional security. Recently, numerous findings with respect to in vitro regeneration and transformation approaches in umpteen rice genotypes have offered an ingenious platform for the nutritional and yield enhancements $[25,26]$. However, predominantly indica rice cultivars around the globe, persist to be unpliable to genetic improvements owing to their exiguous regeneration potential. The existent protocols for regeneration and transformation of indica rice have emerged as arduous, protracted, and stringently genotype-specific with decumbent transformation efficiencies [21, 22]. The indica rice genotypes are invariably recalcitrant to tissue culture and callus induction \& its regeneration involves utilization of genotype-dependent differential media combinations. Hence, implementing any one optimal hormonal-media therapy for triggering callus formation \& its efficient regeneration across of indica rice genotypes is an enormously tedious task. The development of a genotype-free, in vitro regeneration \& plant transformation technique provides a crucial platform for the improving crop breeding performances via rice genome modification employing the CRISPR/Cas 9 approach. Taking into account the implication of plant transformation in translational genomics and crop genetic improvement, we devised a robust, reproducible, universally adaptable and inordinately dynamic transformation and regeneration protocol for various indica rice genotypes to overcome the unwarranted genotype-dependent optimization. We established a genotype-free, replicable method for biolistic-mediated transformation of indigenous indica rice (Oryza sativa L.) genotypes namely, Samba Mahsuri (SM) that is widely cultivated in southern belt of India comprising of 1-2 million hectares of Telangana, Andhra Pradesh, Tamil Nadu and Karnataka as well as Orissa, Bihar and UP, and White Getu (WG), as well as Hamilton (HT) with inherent salt tolerance, employing mature seed-derived calli. We earmarked the elite cultivar or mega-variety i.e. Samba Mahsuri (SM) (BPT 5204) for optimization of different parameters crucial for invoking improved transformation and regeneration frequencies. In order to devise a universal protocol for transformation and regeneration and for the revival of indica rice cultivars of Sundarbans, the standardized protocol was extrapolated to these salt tolerant genotypes WG and HT that catered to the agrarian community in the low-lying islands of Sundarbans, but were overnight lost to the tropical cyclone Aila that ravaged West Bengal in 2009.

\section{Overview of an innovative genotype-free indica rice transformation protocol}

Plant transformation entails few principle steps for instance, embryogenic calli generation, regeneration of shoots and induction of roots, however optimization of all these steps is requisite to suit individual plants. To develop a standard reproducible transformation protocol for rice (Fig. 1), we examined the effects of different combinations of growth regulators on callus- and shoot- regeneration by modifying existing protocol described by Kaul et al. [19]. Findings have been delineated under the following sections.

\section{Induction of Embryogenic calli (EC) in mature seed explant}

In order to obtain EC, we employed sterilized mature seeds as explants. First and foremost, step for a successful plant transformation technique is to obtain sterile or microbial contamination-free cultures. In the present investigation, explant disinfection was performed in three indica rice genotypes. A two-step sterilization process was employed to disinfect the seeds. Firstly, seeds were immersed in $70 \%$ ethanol for 2 min, followed by immersion in $2 \%$ sodium hypochlorite for $18 \mathrm{~min}$ and finally washed by sterile $\mathrm{H}_{2} \mathrm{O}$ (Additional file 1: Table S1). A low rate of seed contamination was observed in all varieties (SM=1\%, WG $=1 \%$, WG $=2 \%$ ) after cultured on callus induction media. (Additional File1: Table S1).

Varying types of explants were employed for induction of EC for instance, leaf base; leaf sheath cells; root; immature- and mature seed-derived embryos. Amongst all, mature seed-derived embryos have been enormously utilized in rice in vitro culture studies [19, 27]. The efficacy of callogenesis and regeneration from explants tissues is influenced not only by the type of explants, physiological status of the explant genotype, but also by the suitable culture medium supplemented with different combinations of plant growth hormones [2]. Indica rice seeds mostly developed into non-EC with extremely low regeneration efficiencies and tedious than japonica subspecies as reported previously [5, 27-30]. Hence, to attain enhanced regeneration efficiency in indica rice we utilized mature seed-derived embryos for development of EC from indica rice genotypes (SM, WG, and HT) due to their easy accessibility throughout the year. To analyze the effect of supplementing an auxin \& cytokinin amalgamation on callus induction (Cl), eight different media combinations were designed (Additional file 1: Table S2). Sterilized seeds of the three genotypes were inoculated in two different basal media [Murashige \& Skoog (MS) and Chu N6] containing differential combinations of growth regulators, i.e., 2, 4-D, dicamba, TDZ, glutamine, and other additives. We observed indistinguishably enhanced callus induction frequencies (CIF) when mature seeds of SM, WG \& HT were placed on the two different basal media. Calli initiated invariably from the scutellar region of the embryo and a visible mass was generated within 5-d. Subsequently, the 15-d-old calli were sub-cultured for another 10-d period to obtain compact, nodular, light yellow or off-white coloured EC of 5-7 mm in size (Fig. 2a-b and 3; Additional file 2: Fig. S1: a-d). Significant differences were observed in CIF (\%) when mature seed were cultured on MS- and N6- media supplemented with different combinations of growth regulators. It was revealed that on media containing 2,4-D alone, the percentage of EC was lower (60-75\%) than that generated on 2,4-D with other additives (96-98\%) (Table 1). We observed efficient CIF in all three genotypes on MS (96-98\%) [CIMM7; Fig. 4a; Table 1] and N6 (95\%) [CIMN7; Fig. 4b; Table 2] media when supplemented with 2, 4-D (2.5 mg/L); dicamba (1.5 mg/L); TDZ ( $0.1 \mathrm{mg} / \mathrm{L})$; proline (1000 mg/L); and glutamine ( $2.5 \mathrm{mg} / \mathrm{L})$. As the MS-based medium supplemented with growth hormones revealed a higher CIF (\%) than N6-supplemented medium for EC formation (Additional file 1: Table S3), we chose the MS supplemented media for further experiments. Further, the 25-d-old calli generated on both CIMM7 \& CIMN7 media showed enhanced proliferation and higher fresh weight (Fig. 4c) than those induced on other $\mathrm{Cl}$ media (Table 3). These finding reveals that presence of 2,4-D $(2.5 \mathrm{mg} / \mathrm{L})$ with other additives is conducive for induction of embryogenic callus. In addition, increasing 2,4-D concentrations beyond $2.5 \mathrm{mg} / \mathrm{L}$ reduces the frequency of $\mathrm{Cl}$. During our experimentation, we found that on employing maltose (3\%) as a carbon source, in place of sucrose effectuates higher EC induction. Osmotic status of the callus may be controlled via supplementation of basal media with maltose, which leads to higher induction rates of EC. Amino acids, for instance, proline \& glutamine as media additives 
proved efficacious for the initiation and sustenance of EC. Moreover, inclusion of thidiazuron (TDZ), dicamba, and casein hydrolysate in the culture media led to enhanced somatic embryogenesis. In view of our findings, we recommend CIMM7 (MS- or N6-based media) for genotype-free embryogenic callus induction with no significant variations in the $\mathrm{Cl}$ responses and confers enhanced TE and shoot RF in the selected indica genotypes. Furthermore, this media composition may overcome the much-hyped consequential variations in $\mathrm{Cl} \&$ regeneration frequencies prevalent amongst the rice genotypes. Henceforth, our proposed $\mathrm{Cl}$ composition is a panacea or a consensus common medium, which ensures induction of EC in different Indica rice genotypes, a feat previously far-fetched. Thus, it may be inferred that $\mathrm{Cl}$ is solely dependent on the permutation and combination of the growth hormones employed to supplement the basal media and independent of the genetic potential of the selected genotype. Overall, the statistical significance analysis of the above-mentioned dataset employing one way ANOVA $(\mathrm{p} \leqslant 0.05)$ followed by Tukey HSD $\left(\mathrm{HSD}_{0.5}\right)$ tests demonstrated that CIF efficiency is influenced by the culture medium compositions.

\section{In vitro regeneration with partial desiccation}

The availability of an effective regeneration protocol is quintessential for plant transformation. Present investigation aimed to develop Cas 9 transgenic rice lines via incorporation of a gRNA-free CRISPR/Cas9 vector employing biolistic-mediated transformation. This may serve as a platform for rice genome editing in the presence of the desired gRNA/s. To achieve successful biolistic transformation, a compatible robust reproducible regeneration protocol was developed prior to transformation. It is well known that plant morphogenesis and growth is maintained by proper auxin and cytokinin ratios. In the course of our investigation, we established that diverse growth regulators and their dosage employed in the regeneration medium play a crucial role in modulating the regeneration frequency. In a bid to endow higher regeneration frequencies, we harmonized the ratio and proportion of the growth regulators systematically to effectuate optimal RFs in all the three indica rice cultivars. Hence, we optimized the medium conditions for in vitro shoot regeneration utilizing MS basal media supplemented with varying permutations and combinations of auxins and cytokinins. To achieve the aforesaid objective, we employed different media compositions (RGM 1 to 8; Refer to Additional file 1: Table S4) for regeneration of calli, wherein RGM6: BAP (1.5 mg/L); NAA (0.5 mg/L); TDZ (1.0 mg/L); zeatin $(0.2 \mathrm{mg} / \mathrm{L})$ and proline $(500 \mathrm{mg} / \mathrm{L})$ rendered the highest regeneration efficiency of the calli incubated on it i.e., SM (94\%), WG (92\%) and HT (90\%), respectively (Fig. 5a, Table 4, and Additional file 1: Table S5). Shoot primordial initiated after 7 days of the inoculation of 25-d-old EC on the regeneration medium (Fig.2c; Additional File 2: Fig. S1-e), followed by elongation of shoots (Additional File 2: Fig. S1-f). Regenerated shoots that adequately elongated after four weeks (Fig.2d; Additional File 2: Fig. S1-g) were transferred to the rooting medium (Fig. 2e; Additional File 2: Fig. S1-h). Our results displayed that fortification of RM (RM1-5) with zeatin $(0.2 \mathrm{mg} / \mathrm{L})$ and proline $(500 \mathrm{mg} / \mathrm{L})$ resurrected into a unique combination of growth regulators (BAP, TDZ, zeatin and NAA) termed as RGM6, which conferred a phenomenal boost-up in the regeneration efficiencies of EC, thereby invoking vigorous emergence of multiple in vitro shoots. Hence, the synergistic impact of exogenous zeatin $(0.2 \mathrm{mg} / \mathrm{L})$ and proline $(500 \mathrm{mg} / \mathrm{L})$ in the regeneration media (RGM6) was instrumental in increasing the shoot regeneration potential from rice seed-derived calli via stimulated cell divisions.

In order to evaluate the effect of desiccation on the regeneration efficiency of non-transformed calli, the mature seed embryo-derived calli were subjected to two experimental conditions, i.e., with- ( 24 h, 48 h, 72 h) \& without-desiccation and assayed for differential efficiencies of regeneration (Fig. $5 b$ ). Noteworthily, incorporation of the dehydration or desiccation stress step, promoted somatic embryogenesis \& shoot regeneration frequencies. Amongst all the calli desiccation treatments, the $48 \mathrm{~h}$ desiccation period enhanced regeneration frequency (RF) in the three rice varieties (SM=94\%; WG $=92 \%$; $H T=90 \%)$ in comparison to non-desiccated fresh calli ( $\mathrm{SM}=84 \%$; WG=82\%; HT=80\%) (Additional file 1: Table S6). Therefore, we concluded that the 25-d-old calli, when subjected to $48 \mathrm{~h}$ partial desiccation phase exhibited maximal regeneration frequencies $(90-94 \%)$. The ANOVA ( $\mathrm{p} \leqslant 0.05)$ followed by Tukey HSD test (HSD 0.5 ) test results showed that regeneration efficiency and plantlet regeneration were mostly influences by the media compositions sans genetic potential of the genotypes. Thus, the RGM6 in consonance with partial desiccation of calli proved to be most efficacious in significantly enhancing regeneration efficiencies. Hence, it may be inferred that besides $\mathrm{Cl}$, regeneration efficiencies of $\mathrm{EC}$ are predominantly dependent on the permutation and combination of the growth hormones employed to supplement the basal media and independent of the genetic potential of the selected genotype.

\section{Biolistic transformation and rhizogenesis of transformants}

In vitro regeneration-based plant transformation approaches offer a crucial platform for basic and translational studies in plant science. Partially desiccated mature seed derived EC were the most preferable explants for biolistic transformation due to their higher RF. For efficacious delivery of CRISPR-Cas 9 reagents, we employed an optimized biolistic-mediated transformation approach. Umpteen factors affecting bombardment efficacy were optimized to establish a simple and reproducible technique of transformation in indica genotypes (SM, WG, HT), utilizing the indigenously developed NICTK-1_pCRISPR-Cas9 markerfree vector that harboured the cassette of codon-optimized Cas9 gene (Additional file 1: Table S7). Different factors were optimized for instances, particle dimension $(0.4-1.0 \mu \mathrm{m})$, target distance (3.0-9.0 cm), helium pressure (1,100 psi), and concentration of DNA (1.5-2.5 $\mu \mathrm{g} / \mathrm{shot})$. When EC were shot with gold particles (sized $0.6 \mu \mathrm{m}$ ) carrying $2 \mu \mathrm{g}$ /shot of DNA, employing a helium pressure of $1.100 \mathrm{psi}$ at an appropriate target distance (9 $\mathrm{cm}$ ) generated the highest transformation efficiencies (TE) in indica genotypes (SM, WG, HT) (Fig. 6k; Additional file 1: Table S7).

In addition, significant enhancements in regeneration efficiencies of bombarded calli were observed when pre-incubated onto $\mathrm{Cl}$ media with variable osmotica for instance, mannitol, sorbitol and $\mathrm{AgNO}_{3}$. Calli placed on $\mathrm{Cl}$ media supplemented with mannitol (36g/L), sorbitol ( $\left.36 \mathrm{~g} / \mathrm{L}\right), \& \mathrm{AgNO} 3(5 \mathrm{mg} / \mathrm{L})$-a potent inhibitor of ethylene action for $48 \mathrm{~h}$ prior to bombardment, invigorated regeneration efficiencies $(\mathrm{SM}=88 \%$; $\mathrm{WG}=87 \%$; $\mathrm{HT}=84 \%)$ than those cultured on regeneration media sans supplementation of osmoticum ( $\mathrm{SM}=84 \%$; WG $=82 \% ; \mathrm{HT}=80 \%$ ). Regeneration efficiencies of transformed calli were lower (81-86\%) than non-transformed ones (90-94\%) (Fig 6j; Additional file 1: Table S8). The transformed calli were kept in dark for another 7-d-period on Cl media and then transferred to regeneration media (RM) for induction of in vitro plantlets (previously described in CIMM7 \& RGM6). The plantlets were eventually transferred to a hormone-free MS (half strength) medium for rhizogenesis (Fig. 2e; Additional File 2: Fig. S1-h). Consequently, a high frequency (100\%) of root proliferation was recorded in the three varieties after 7-10 days of transfer. Shoots that attained a height of 3-4 inches and developed adequate roots were hardened in small pots with soil for a week (Additional File 2: Fig. S1-i). The hardened seedlings were further transferred to greenhouse field maintained at controlled temperature and humidity (Fig. 2f; Additional File 2: Fig. S1-j). Globally, numerous plant transformation strategies for instance agrobacterium- and biolistic- 
mediated methods, nanoparticle- and viral vector-based deliveries have been employed to incorporate useful traits for both indica and japonica genotypes [19, $24,31]$. Interestingly, sufficient reports highlight the efficient recovery of transgenic indica rice plants from calli utilizing the biolistic approach. Remarkably, our protocol emerges as a crucial intervention in elevation of the indica rice transformation efficiencies to a great extent (65-69\%) than previously reported figures (47\%) [32].

\section{Molecular screening and validation of transform plantlets}

In recent past, numerous reports have demonstrated the differential applications of CRISPR/Cas- based genome editing in plants. The genome edited plants have been interrogated employing multiple screening techniques to validate the indels or mutations, thereby incorporating the desired traits [7, $8,9,11,12,22$, 26]. We elucidate the development of transgenic plants in recalcitrant indica rice genotypes via transferring the CRISPR/Cas9 reagents into rice genome employing biolistic approach. In order to validate if exogenous Cas9 gene was integrated into the rice genome, genomic PCR analysis of the transgene in transgenic lines was performed. Genomic DNA amplification of a Cas 9 gene fragment sized $531 \mathrm{bp}$ was observed in the putatively transformed $\mathrm{T}_{0}$ plants in comparison to wild-type (WT) (Fig. 6a-c; Additional File 2: Fig. S2:a). PCR analyses of transgenic lines revealed that the highest transformation efficiency was achieved in SM (69\%) in comparison to WG (67\%), and HT (65\%) (Additional file 1: Table S9). Incidentally, the TE reported previously in SM was recorded as low as $47 \%$ employing agro-mediated transformation by Reddy et al. [32]. Contrastingly, our protocol highlights a significant enhancement in transformation efficiency (69\%) in this SM indica rice genotype. Moreover, a minimal TE of $6.5 \%$ was obtained via the particle-bombardment approach reported by Cho et al. [33]. Notably, this is the first report to establish a robust regeneration, and transformation protocol for salt tolerant varieties, i.e., White Getu and Hamilton. Indeed our ingenious protocol offers a robust, suitably optimized, genotype-free, universally applicable transformation strategy that may be effectively extrapolated to any rice cultivar, globally.

Subsequently, we performed digoxygenin (DIG)-based Southern blot analysis employing a Cas9-specific probe to ascertain the number of transgene integration sites for the PCR positive lines (Fig. 6g-i; Additional File 2: Fig. S2-b). The gDNAs of selected PCR positive transgenic plants from each genotype and their respective WT plants were digested with the restriction enzyme EcoRV as a single restriction site occurred in Cas 9 gene. After hybridization with a non-radioactively labeled Cas9 gene-specific probe followed by autoradiography, varying sizes of hybridization signals or bands that corresponded to Cas 9 gene fragments were observed (Fig. 6g-i; Additional File 2: Fig. S2-b). Most of the plants revealed a single copy of Cas 9 gene insertion and two out of 18 transgenic lines revealed one to two copy insertions, thereby demonstrating stable transgene integration into the rice genomes (SM, WT, HT). On the contrary, no bands or signals were detected in WT lanes. Southern blot signals substantiate the Cas 9 gene copy number in the transgenic plants as independent events. Based on the Southern analysis, we inferred that the PCR positive transgenic lines were independent transgenic events. Further, the PCR and Southern positive lines from each genotype were validated via Sanger's sequencing (Fig. 6d-f; Additional File 3: Fig. S1-S6). Selected transgenic $T_{0}$ plants were selfed, and seeds of individual lines were collected and subsequently germinated upon MS (half-strength) medium for further analysis. Upon germination, the $\mathrm{T}_{1}$ seedlings were PCR analyzed for the presence of Cas 9 transgene employing gene specific primers. Results showed that the targeted Cas 9 gene was inherited with a 3:1 Mendelian ratio for single-copy insertions (Additional file 1: Table S10). Hence, results suggested that Cas9 transgene was stably delivered to subsequent generations.

\section{Phenotype of transgenic rice lines}

In a bid to determine if the presence of Cas 9 transgene would affect the morphology of the transgenic plants, we monitored their phenotypes. Mature seedderived in vitro WT and transgenic $T_{1}$ plants of the three genotypes were allowed to grow in field under controlled temperature and humidity conditions, to be sampled randomly. Their morpho-agronomic trait performances, for instance, plant height, leaves length \& width, tiller numbers, number of productive panicles, length of panicles, filled grains/spike, 1000 grains weight, and so on were examined. We observed that in vitro regenerated WT and transformed plants were phenotypically indistinguishable and revealed better performances than WT (Additional file 1: Table S11). The Cas9 transgenic rice lines did not display any morphological variations in comparison to the WT (Table 5 and Additional file 2: Fig. S3-S5). In addition, the enhanced number of filled grains or spikes were obtained in SM, WG \& HT transgenic lines, which revealed sustainably good agronomic performances (Additional file 1: Table S12). Moreover, the weight of 1000 grains revealed in the order of highest to lowest in the three genotypes was $S M>W G>H T$ (Fig. 7). Furthermore, at maturity, all the confirmed transgenic rice lines were fertile and exhibited normal phenotype. Subsequently, we recorded the agronomic trait performances for transgenic and WT plants under simulated field conditions and astoundingly the transgenic lines of SM, WG, HT showed high yield potentials (Fig.7). Taken together, results strongly suggested that SM, WG \& HT varieties responded efficaciously to the devised plant transformation protocol that may be utilized for further improvement of their nutritional and yield potentials via CRISPR/Cas9-based gene editing approaches.

In addition, we notably attribute the robustness of our method to few critical factors; for instance, highly efficient mature seed explant; improvement in CIF by addition of maltose (carbon source), proline \& glutamine (amino acids), enhancement in RF with partial desiccation approach; biolistic-mediated DNA delivery approach, post-bombardment recovery on regeneration media and transgenic rice lines displaying efficient integration of Cas9 gene into the rice genome. The protocol presented here has significant advantages over the methods currently available especially in recalcitrant indica rice cultivars, for instance, higher CIF, large number of regenerated transgenic plants per calli within a short time span, stable transfer of transgene copy in subsequent generation. This novel protocol (Fig.1) offers an efficacious, viable, genotype-free strategy for genome editing in indica rice, in order to introduce agronomically important traits. Undoubtedly, our innovative protocol offers a robust platform that may be universally adaptable transformation strategy rapidly extrapolated to all rice cultivars, globally.

\section{Conclusion}


Strategies involving genetic transformation are quintessential for interrogation of gene functions as well as for introduction of novel agronomic traits. Undoubtedly, rice (Oryza sativa L.) has emerged as the prime cereal crop that feeds more than half of the global population. Taking cognizance of the intricacies and inherent constraints that we encounter during callus induction, de-differentiation \& transformation of recalcitrant indica rice cultivars, an effortlessly efficacious, reproducible and genotype-independent protocol for indica rice transformation was developed that introduced the Cas 9 gene into the rice genome through particle bombardment. We optimized the concinnity amongst the significant factors that influence the callus induction \& regeneration responses via orchestrating the growth regulators- auxins \& cytokinins, in order to effectuate an accentuated regeneration efficiency \& transformation frequency in indica rice. Furthermore, amid the list of prominent factors impacting the in vitro regeneration \& transformation in indica rice, we highlighted that incubating the callus in dark along with partial desiccation and fine-tuning the parameters related to bombardment, culminated into significant improvement in these processes. The current strategy encompasses a series of steps that equips a researcher in the field of rice crop improvement with an efficacious genotype-free method to achieve enhanced CIF (98\%), RF (94\%) and TE (69\%). Intriguingly, the regeneration \& transformation efficiencies of the three abovementioned rice cultivars (SM, WG, HT) were astoundingly indistinguishable. Additionally, amongst the three rice cultivars employed in this study, WT \& HT are innately salt tolerant. Hence, introduction of salt resistance in these cultivars via genome editing would ensure their increased adaptability, viability \& productivity in coastal areas of our country. Moreover, this protocol is time-efficient and highly productive that may be employed as a potent and ultra-effective tool to generate genome-edited indica rice lines with crucial agronomic traits for instance, high yield, more nutrition \& resistance to stress within minimal time span.

\section{Materials And Methods}

\section{Plant material and explant preparation}

Mature, healthy dry rice (Oryza sativa L.) seeds of Samba Mahsuri, White Getu, and Hamilton were used as explants in the present investigation. These seeds were procured from the diverse regions of India. Seeds were manually dehusked and surface sterilized with $70 \%$ ethanol for 2 min. The seeds were rinsed with sterilized distilled water (thrice) to remove ethanol traces. Subsequently, these seeds were immersed in a mixture of $2 \%$ sodium hypochlorite and Tween 20 $(100 \mu \mathrm{l})$ for $18 \mathrm{~min}$, with continuous shaking. Later, the seeds were rinsed and air-dried on sterile tissue paper. Finally, these seeds were placed in CIM.

\section{Embryogenic $\mathrm{Cl}$ and proliferation}

Sterilized seeds (8-10 seeds/plate) were inoculated on MS basal [34] (Hi-Media, Mumbai, India) and CHU-N6 medias [35], supplemented with maltose (30 g/L), glucose $(10 \mathrm{~g} / \mathrm{L})$, casein hydrolysate $(0.3 \mathrm{~g} / \mathrm{L})$, amino acid: proline (0.5-1.0 mg/L), glutamine (1.0-3.0 mg/L) and hormones: 2,4-D (1.5-3.0 mg/L), TDZ (0.1-0.2 $\mathrm{mg} / \mathrm{L})$, dicamba $(0.5-2.0 \mathrm{mg} / \mathrm{L})$ and phytagel gelling agent $(4.0 \mathrm{~g} / \mathrm{L})$ and set at $5.8 \mathrm{pH}$. Different media compositions were ascertained as, CIMM 1-8 (with $\mathrm{MS}$ as basal medium) and CIMN1-8 (with N6 salt basal medium) (Additional file 1: Table S2). After 5-d-incubation period in dark $\left(\mathrm{Tm}=25 \pm 2{ }^{\circ} \mathrm{C}, \mathrm{RH}=50-60 \%\right.$ ) roots emerged that were excised for induction of healthy callus. 5-d-old minuscule calli arising from the scutellar zone of endosperm were detached and incubated on a fresh $\mathrm{Cl}$ medium with a similar environmental condition for an extended period of 10 days, prior to biolistic transformation. The embryogenic calli thus obtained were kept for $48 \mathrm{~h}$ (dark, Tm=27 $\left.\pm 1^{\circ} \mathrm{C}, \mathrm{RH}=50-60 \%\right)$ in osmotic medium: mannitol (36g/L); sorbitol (36g/L); $\mathrm{AgNo} 3(5 \mathrm{mg} / \mathrm{L}) ;$ sucrose ( $3 \%$ ) for a period of $48 \mathrm{~h}$. The experiments were performed in triplicate and CIF was recorded by the formula:

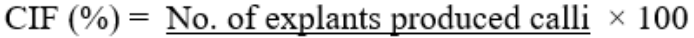

$$
\text { No. of cultured explants }
$$

\section{Partial desiccation and in vitro regeneration}

The EC (25-d-old) were transferred onto sterilized Whatman filter paper ( $3 \mathrm{~mm})$ placed in petri-dish \& sealed by parafilm. The desired amount of desiccation was achieved by keeping EC for varying time points $(24,48$, and $72 \mathrm{~h})$ in the growth chamber under controlled temp. $\left(27 \pm 1{ }^{\circ} \mathrm{C}\right) \& \mathrm{humidity}(50-60 \%)$. Following partial desiccation, calli with- and without-desiccation were transferred to MS containing shoot induction media (RGM 1 to 8 ) supplemented with auxins: BAP (0.5-2.0 mg/L), TDZ (0.2-1.5 mg/L), \& zeatin (0.5-1.0 mg/L) and cytokinins: NAA (0.5-1.0 mg/L) and amino acid: proline (0.3-0.5mg/L) as mentioned in (Additional file 1: Table S4). For shoot induction, calli (approximately 10) were placed on RM in culture bottles that in turn were kept in the growth chamber ( Tm $=25 \pm 2{ }^{\circ} \mathrm{C}$, light/dark: 6/8 h, 2000 lux) for a week. The experiment was replicated thrice and the sub-culturing was performed after 7-d intervals in the same particular media amalgamations for sufficient shoot elongation. Data of plant regeneration efficiency was recorded post 30-d-period by calculating the efficient number of shoots induced from the given cultured calli using the following formula-

\section{$\mathrm{RF}(\%)=\underline{\text { No. of plants regenerated }} \times 100$}

\section{No. of calli inoculated}

\section{Rhizogenesis, transplantation, and acclimatization for field establishment}

Well-developed shoots were excised and inoculated onto hormone-free MS (half-strength) media solidified with phytagel (3\%). Those culture bottles were placed in growth chamber maintained under controlled temp, $\mathrm{RH}$ \& light conditions $\left(\mathrm{Tm}=27 \pm 2^{\circ} \mathrm{C}\right.$; $\left.\mathrm{RH}=50-60 \%\right)$ for induction of roots. Plantlets with profuse rooting system were excised carefully from the medium within culture bottles and transplanted to plastic pots with soil: sand: vermiculite (1:1:1) mixture for 10-d-period of hardening. For the proper growth of plantlets, the pots were covered completely by a transparent polythene bag bearing minuscule perforations to enable air circulation. After the 10-d hardening phase, the polythene bags were removed and the plantlets were transplanted to the earthen pots containing 
sand: soil: compost (1:3:1) mixture and placed in greenhouse under controlled temp \& humidity conditions $\left(\mathrm{Tm}=28 \pm 2^{\circ} \mathrm{C}\right.$; $\left.\mathrm{RH}=85 \%\right)$. Finally, when new leaves emerged, the plants were transplanted into the field.

\section{CRISPR/Cas9 vector construction for biolistic transformation}

A robust marker-free pCAMBIA1300-based plant expression vector NICTK-1_pCRISPR-Cas9 (16.0 kb) was designed indigenously that harbours a 6.6 kb of transgene cassette of rice plant codon optimized SpCas9 gene (4.1 kb), where nuclear localization signals (NLSs) are attached at both ends with high GCcontent at the $5^{\prime}$ end The Cas 9 gene expression cassette comprised of Zea mays ubiquitin (pUbi) promoter (1.9 kb) along with nopaline synthase (NOS) (253 bp) as the terminator was flanked by Srf1-Srf1 restriction sites. The vector consisted of four multiple cloning sites, i.e., Bsal-Bsal; Swa1-Sbf1; Swa1-ASiSl; Sbf1ASiSI, which can be utilized for the integration of single or assembly of multiplexed sgRNAs for introduction of one or more agronomically important traits in plants (Fig. 8 and Additional file 3: Fig. S6).

\section{Transformation of rice employing biolistic approach}

The plasmid of the indigenously designed NICTK-1_pCRISPR-Cas9 plant transformation vector that harboured the Cas 9 expression cassette was isolated by alkaline lysis method, according to

Sambrook et al. [36]. The biolistic transformation was performed using the Helium-powered Particle Delivery System (PDS1000/He) from Biorad. In order to systematize factors influencing callus transformation, we tweaked few parameters related to the particle bombardment process, for instance, verified the helium pressure (110-1600 psi), fine-tuned the distance of the callus from the stopping screen (3.0-9.0 cm), a gap distance (1/2-1/4 inch), macrocarrier flight

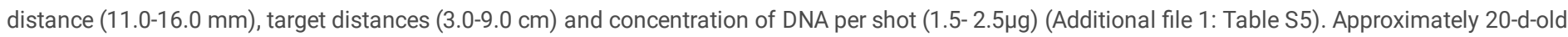
embryogenic calli were placed in the center of the petri plate containing $\mathrm{Cl}$ media for better bombardment. A suspension of gold particles $(0.6 \mu \mathrm{m})$ was prepared prior to the transformation process; afterwards, the plasmid DNA harboring the Cas 9 expression cassette was coated upon the gold particles as described by Kaul et al. [19]. Further, the calli on pre- existing petri-dish containing CIM were kept in the dark for $7 \mathrm{~d}$ period for post bombardment recovery. As the calli enlarged they were transferred to shoot induction media containing petri-dishes (previously described media composition). Once shoots emerged from the calli, these were rooted in MS (half strength) media that led to developed healthy plantlets. These putatively transformed seedlings/plantlets from bombarded calli were maintained greenhouses under controlled temperature \& humidity condition. Cas 9 gene integration was validated by PCR and Southern analysis.

\section{Molecular validation of transgene integration}

Total genomic DNA (gDNA) was extracted from leaf tissues of putatively transformed and WT plants employing a modified CTAB protocol [37]. The gDNA was utilized as template for PCR analysis. Putative $T_{0}$ transgenic rice lines harbouring CRISPR/Cas9 construct were validated by PCR analysis using Cas 9 gene specific primers (Cas9F: 5' TTCGACCAGTCCAAGAACGG 3'; Cas9R: 5' CTTGACCTTGGTGAGCTCGT 3'). PCR reaction was performed using conditions as follows: an initial denaturation at $95^{\circ} \mathrm{C}$ for $5 \mathrm{~min}$. followed by 30 cycles of denaturation at $94^{\circ} \mathrm{C}\left(1 \mathrm{~min}\right.$.); annealing at $58^{\circ} \mathrm{C}\left(1 \mathrm{~min}\right.$.); and for extension at $72^{\circ} \mathrm{C}$ (30 s) with final extension at $72^{\circ} \mathrm{C}(10 \mathrm{~min}$.) (Agilent gradient thermocycler, Sure cycler 8800$)$. The reaction was accompanied by positive \& negative controls employing Cas9 harbouring plasmid \& gDNA plasmid from WT, respectively. The PCR amplified products were separated on agarose gel (0.8\%) and visualized by gel documentation unit (Alpha Imager EP). Further, confirmation of the stable transgene (Cas9) integration into the $\mathrm{T}_{1}$ was performed by, $\mathrm{PCR}$ analysis carried out according to the above-mentioned PCR conditions. PCR-positive Cas9 lines were further validated by southern blot analysis (non-radioactive method) according to the method described by Kaul et al. [19]. In brief, the gDNAs (10 $\mu \mathrm{g})$ from both Cas 9 transgenic and WT plants were digested with the ECoRV and electrophoresed on agarose gel (1.0\%). The gel with digested gDNAs was blotted on to positively charged nitrocellulose membrane (Hybond-N+, Amersham, UK) and hybridized with a non-radioactively labeled Cas9 PCR fragment as a probe following manufacturer's instructions (Digoxigenin: Roche, DIG DNA Labelling and Detection Kit, version 19, 2004). For synthesis of the probe, PCR amplified product (Cas9) was labeled with Digoxigenin (DIG; Roche, DIG DNA Labelling and Detection Kit, version 19, 2004). Additionally, ECoRV digested CRISPR/Cas9 vector plasmid and the undigested gDNA of WT plant were used as positive and negative controls, respectively.

Eventually, Cas9 positive PCR products were gel purified using gel purification kit (QIAQuick Gel Extraction Kit) and used for automated sequencing (Macrogen). Obtained sequences were analyzed through NCBI-BLAST programme.

The TE was recorded using the following formula:

\section{$\mathrm{TE}(\%)=\underline{\text { No. of putative positive plants }} \times 100 \%$}

\section{No. of calli used for biolistic transformation}

\section{Agronomic trait performances}

Phenotypic performances of the seed- \& in vitro-derived WT and transgenic plants were monitored under simulated field conditions in greenhouse maintained at controlled temperature \& humidity conditions. Plants were scored for different agronomic trait performances, for instance, plant height, number of tillers and panicles, flag leaf length, root length, spikelet density, yield potentiality, and others.

\section{Statistically data analysis}


All experiments in terms of callus induction frequency, regeneration frequency, and transformation efficiency were conducted in triplicate with three independent biological replicates. Data was analyzed statistically via one-way analysis of variance (ANOVA) using a complete randomized design. The groups that showed variance were then subjected to Tukey HSD test $\left(\mathrm{HSD}_{0.5}\right)$ Duncan's Multiple Range Test 10 with a significance value of $p \leqslant 0.05$.

\section{Abbreviations}

CRISPR: Clustered Regularly Interspaced Short Palindromic Repeats

PCR: Polymerase chain reaction

DNA: Deoxyribonucleic acid

mg: Milligrams

g: Grams

L: Litres

DIG: Digoxigenin

ANOVA: One Way Analysis of Variance

NAA: 1-Naphthalene Acetic Acid

BAP: 6-Benzylaminopurine

TDZ: Thidiazuron

2,4-D: 2,4-Dichlorophenoxyacetic acid

\section{Declarations}

Ethics approval and consent to participate

Not applicable.

\section{Consent for publication}

Not applicable.

\section{Competing interests}

The authors declare that they have no competing interests.

\section{Availability of data and materials}

Data generated or analyzed during this study has been comprehensively included in this published article.

\section{Funding}

This work was supported by Grants received from the ICAR-National Agricultural Science Fund (NASF - Grant F. No. NASF/GTR- 7025/2018-19) for funding this research.

\section{Authors Contribution}

TK devised the research plan and design the manuscript. TK and SKS provided critical inputs in designing the NICTK-1-pCRISPR-Cas9 vector. SKS and KHF participated in the experiment. SKS wrote the first draft of manuscript. MN was involved in data analysis. JB and RV modified the manuscript. AT and RK helped in molecular analysis of transgenic plants. ME participated in figure preparations. All authors read and approved the final manuscript.

\section{Acknowledgements}

The authors thank the ICAR scheme-National Agricultural Science Fund (NASF/GTR-7025/2018-19) for financially supporting this research. We also thank the ICGEB-Core Funds for additional support towards the completion of this work.

\section{Authors' information}

${ }^{1}$ Nutritional improvement of Crops Group, International Centre for Genetic Engineering and Biotechnology (ICGEB), New Delhi, India.

\section{References}


1. Kumari R, Sharma VK, Kumar H. Seed culture of rice cultivars under salt stress. Int J Pure Appl Biosci. 2015;3:191-202.

2. Mostafiz SB, Wagiran A. Efficient callus induction and regeneration in selected Indica rice. Agronomy. 2018;8:77.

3. Tie W, Zhou F, Wang L, Xie W, Chen H, Li X. Reasons for lower transformation efficiency in indica rice using Agrobacterium tumefaciens-mediated transformation: lessons from transformation assays and genome-wide expression profiling. Plant Mol Biol. 2012;78(1-2):1-18.

4. Savary S, Willocquet L, Elazegui FA, Castilla NP, Teng PS. Rice pest constraints in tropical Asia: quantification of yield losses due to rice pests in a range of production situations. Plant Disease. 2000;84(3):357-369.

5. Govindaraj M, Vetriventhan M, Srinivasan M. Importance of Genetic Diversity Assessment in Crop Plants and Its Recent Advances: An Overview of Its Analytical Perspectives. Genetics Res Int. 2015:1-14.

6. Jaganathan D, Ramasamy K, Sellamuthu G, Jayabalan S, Venkataraman G. CRISPR for Crop Improvement: An Update Review. Front Plant Sci. 2018;9:985.

7. Kaul T, Raman NM, Eswaran M, Thangaraj A, Verma R, Sony SK, Sathelly KM, Kaul R, Yadava P, Agrawal PK. Data mining by pluralistic approach on CRISPR gene editing in plants. Front Plant Sci. 2019;10:801.

8. Raman NM, Eswaran M, Bharti J, Motalb KFA, Verma R, Kaul R, Kaul T. Ushering in CRISPR/Cas mediated genome engineering for crops. Sch Acad J Biosci. 2019;7:313-320.

9. Dong OX, Yu S, Jain R, Zhang N, Duong PQ, Butler C, Li Y, Lipzen A, Martin JA, Barry KW, Schmutz J, Tian L, Ronald PC. Marker-free carotenoid-enriched rice generated through targeted gene insertion using CRISPR-Cas9. Nat Comm. 2020;11(1):1178.

10. Sandhya D, Jogam P, Allini VR, Abbagani S, Alok A. The present and potential future methods for delivering CRISPR/Cas 9 components in plants. J Genet Eng Biotechnol. 2020;18(1):25.

11. Kaul T, Sony SK, Raman NM, Eswaran M, Verma R, Thangaraj A, Bharti J, Motelb KFA, Kaul R. How crisp is CRISPR? CRISPR/Cas mediated crop improvement with special focus on nutritional traits; in Advancement in Crop Improvement Techniques (eds) N Tuteja, R Tuteja, N Passricha, S Saifi (New Delhi: Woodhead Printing) 2020;20:159-197.

12. Kaul T, Sony SK, Verma R, Motelb KFA, Thangaraj A, Eswaran M, Bharti J, Nehra M, Kaul R. Revisiting CRISPR-Cas mediated crop improvement:special focus on nutrition. J Biosci. 2020;45:137.

13. Wang F, Wang C, Liu P, Lei C, Hao W, Gao Y, Liu Y, Zhao K. Enhanced Rice Blast Resistance by CRISPR/Cas9-Targeted Mutagenesis of the ERF Transcription Factor Gene OsERF922. PLoS ONE. 2016;11(4):e0154027.

14. Li J, Meng XB, Zong Y, Chen KL, Zhang HW, Liu JX, Li JY, Gao CX. Gene replacements and insertions in rice by intron targeting using CRISPR-Cas9. Nat Plants. 2016;2:16139.

15. Li C, Zong Y, Wang Y, Jin S, Zhang D, Song Q, Zhang R, Gao C. Expanded base editing in rice and wheat using a Cas9-adenosine deaminase fusion. Genome Biol. 2018;19:59.

16. Mishra R, Joshi RK and Zhao K. Genome Editing in Rice: Recent Advances, Challenges, and Future Implications. Front Plant Sci. 2018;9:1361.

17. Endo M, Toki S. Genome editing in rice. Rice 2020;13:27.

18. Ge X, Chu Z, Lin Y, Wang S. A tissue culture system for different germplasms of indica rice. Plant Cell Rep. 2006;25:392-402.

19. Kaul T, Sony SK, Raman NM, Motelb KFA, Bharti J. Genotype-Independent Regeneration and Transformation Protocol for Rice Cultivars. In: Bandyopadhyay A., Thilmony R. (eds) Rice Genome Engineering and Gene Editing. Methods in Mol Biol. 2021; 2238. Humana, New York, NY.

20. Yaqoob U, Kaul T, Nawchoo IA. Development of an efficient protocol for Agrobacterium mediated transformation of some recalcitrant indica rice varieties. Indian J Plant Physiol. 2017;22(3):346-353.

21. Datta K, Datta SK. Indica Rice (Oryza sativa, BR29 and IR64). In: Wang K. (eds) Agrobacterium Protocols. Humana Press. Methods Mol Biol. $2006 ; 343$.

22. Yadava P, Abhishek A, Singh R, Singh I, Kaul T, Pattanayak A, Agrawal, PK. Advances in Maize Transformation Technologies and Development of Transgenic Maize. Front. Plant Sci. 2017;7:1949.

23. Li J, Meng XB, Zong Y, Chen KL, Zhang HW, Liu JX, Li JY, Gao CX. Gene replacements and insertions in rice by intron targeting using CRISPR-Cas9. Nat Plants. 2016;2:16139.

24. Christou P, Ford T, Kofron M. Production of transgenic rice (Oryza sativa L.) plants from agronomically important indica and japonica varieties via electric discharge particle acceleration of exogenous DNA into immature zygotic embryos. Bioflech. 1991;9:957-962.

25. Das P, Adak S, Lahiri MA. Genetic Manipulation for Improved Nutritional Quality in Rice. Front Genet. 2020;11.

26. Liang Y, Biswas S, Kim B, Bailey-Serres J, Septiningsih EM. Improved Transformation and Regeneration of Indica Rice: Disruption of SUB1A as a Test Case via CRISPR-Cas9. Int J Mol Sci. 2021;22(13):6989.

27. Hiei Y, Komari T. Agrobacterium-mediated transformation of rice using immature embryos or calli induced from mature seed. Nat Protoc. 2008;3:824-834.

28. Mallikarjuna G, Mallikarjuna K, Reddy MK, Kaul T. Expression of OsDREB2A transcription factor confers enhanced dehydration and salt stress tolerance in rice (Oryza sativa L.) Biotechnol Letters. 2011;33(8):1689-1697.

29. Yaqoob U, Kaul T, Nawchoo IA. In vitro plant regeneration of some recalcitrant indica rice (Oryza sativa L.) varieties. Vegetos. 2021;34:102-106.

30. Hiei Y, Komari T. Improved protocols for transformation of indica rice mediated by Agrobacterium tumefaciens. Plant Cell Tissue and Organ Cult. 2006;85:271-283.

31. Mukhtar Z, Hasnain S. Optimization of particle bombardment conditions for rice (Oryza sativa L.) Transformation. Pak J Agri Sci. 2018;55(2):271-278.

32. Reddy SSS, Singh B, Peter AJ, Venkateswar RT. Production of transgenic local rice cultivars (Oryza sativa L.) for improved drought tolerance using Agrobacterium-mediated transformation. Saudi J Biol Sci. 2019;26(5):1093 
33. Cho MJ, Yano H, Okamoto D, Kim HK, Jung HR, Newcomb K, Le VK, Yoo HS, Langham R, Buchanan BB, Lemaux PG. Stable transformation of rice (Oryza sativa L.) via microprojectile bombardment of highly regenerative, green tissues derived from mature seed. Plant Cell Rep. 2004;22:483-489.

34. Murashige T, Skoog FA. Revised medium for rapid growth and bio assays with tobacco tissue cultures. Physiol Plant. 1962;15:473-497.

35. Chu CC, Wang CC, Sun CS, Hsu C, Yin KC, Chu CY, Pi FY. Establishment of an efficient medium for anther culture of rice through comparative experiments on the nitrogen sources. Sci Sin. 1975;18:659-668.

36. Sambrook J, Fritsch EF, Maniatis T Molecular cloning: a laboratory manual. Cold Spring Harbor Laboratory Press, Cold Spring Harbor, N.Y. 1989.

37. Doyle JJ, Doyle JL. A rapid DNA isolation procedure from small quantities of fresh leaf tissues. Phytochem Bul. 1987;19:11-15.

\section{Tables}

Table 1 Effect of different plant growth regulators in combination with MS-based medium on callogenesis.

\begin{tabular}{|c|c|c|c|c|}
\hline \multirow[t]{2}{*}{ Media } & \multirow[t]{2}{*}{$\mathrm{D}_{\mathrm{ci}}$} & \multicolumn{3}{|c|}{ Callus induction frequency (\%) } \\
\hline & & SM & WG & HT \\
\hline CIMM1 & 11 & $60.39 \pm 0.679^{a c}$ & $75.49 \pm 0.849^{b}$ & $60.37 \pm 0.641^{\mathrm{ac}}$ \\
\hline CIMM2 & 11 & $80.33 \pm 0.577^{a}$ & $71.80 \pm 0.329^{b}$ & $75.47 \pm 0.441^{c}$ \\
\hline CIMM3 & 9 & $73.09 \pm 0.211^{a}$ & $75.98 \pm 0.849^{b}$ & $78.53 \pm 0.660^{c}$ \\
\hline CIMM4 & 7 & $65.95 \pm 0.063^{a}$ & $74.59 \pm 0.840^{b}$ & $69.06 \pm 0.301^{c}$ \\
\hline CIMM5 & 8 & $74.48 \pm 0.501^{a}$ & $80.21 \pm 0.951^{\mathrm{b}}$ & $72.48 \pm 0.470^{c}$ \\
\hline CIMM6 & 7 & $82.73 \pm 0.339^{a}$ & $84.90 \pm 0.169^{b}$ & $68.48 \pm 0.450^{c}$ \\
\hline CIMM7 & 5 & $98.55 \pm 0.342^{\mathrm{abc}}$ & $97.43 \pm 0.783^{a b c}$ & $96.23 \pm 0.104^{a b c}$ \\
\hline CIMM8 & 8 & $81.7 \pm 0.674^{\mathrm{a}}$ & $87.925 \pm 0.128^{b}$ & $68.08 \pm 0.763^{c}$ \\
\hline
\end{tabular}

$D_{c i}$ : Days to callus initiation

Farequency (mean $\pm \mathrm{SE}$ ) was calculated as the ratio of the number of embryogenic calli to the total number of explant used. Three independent experiments with three replicates each were carried out for each cultivar, i.e., SM, WG, and HT.

Values followed by the same superscript letters are not significantly different at $P=0.05$ according to the ANOVA test

Table 2 Callus induction frequency (\%) in $\mathrm{N}_{6}$ - based medium supplemented with different growth hormones.

\begin{tabular}{lllll} 
Media & $D_{c i}$ & \multicolumn{3}{c}{ Callus induction frequency (\%) } \\
\cline { 3 - 5 } & & SM & WG & $H T$ \\
\hline CIMN1 & 11 & $64.56 \pm 0.513^{\mathrm{a}}$ & $62.67 \pm 0.779^{\mathrm{b}}$ & $68.79 \pm 0.080^{\mathrm{c}}$ \\
\hline CIMN2 & 11 & $79.66 \pm 0.577^{\mathrm{a}}$ & $81.95 \pm 0.929^{\mathrm{b}}$ & $72.05 \pm 0.798^{\mathrm{c}}$ \\
\hline CIMN3 & 9 & $72.72 \pm 0.433^{\mathrm{abc}}$ & $72.23 \pm 0.622^{\mathrm{abc}}$ & $71.93 \pm 0.326^{\mathrm{abc}}$ \\
\hline CIMN4 & 7 & $60.61 \pm 0.531^{\mathrm{a}}$ & $80.12 \pm 0.816^{\mathrm{b}}$ & $70.19 \pm 0.767^{\mathrm{c}}$ \\
\hline CIMN5 & 8 & $75.60 \pm 0.626^{\mathrm{ac}}$ & $78.03 \pm 0.917^{\mathrm{b}}$ & $74.48 \pm 0.501^{\mathrm{ac}}$ \\
\hline CIMN6 & 7 & $87.87 \pm 0.763^{\mathrm{a}}$ & $82.61 \pm 0.339^{\mathrm{b}}$ & $80.33 \pm 0.577^{\mathrm{c}}$ \\
\hline CIMN7 & 5 & $95.42 \pm 0.277^{\mathrm{abc}}$ & $95.67 \pm 0.742^{\mathrm{abc}}$ & $95.03 \pm 0.898^{\mathrm{abc}}$ \\
\hline CIMN8 & 8 & $82.79 \pm 0.500^{\mathrm{a}}$ & $89.33 \pm 0.577^{\mathrm{b}}$ & $87.03 \pm 0.731^{\mathrm{c}}$
\end{tabular}

$\mathrm{D}_{\mathrm{ci}}$ : Days to callus initiation

Farequency (mean \pm SE) was calculated as the ratio of the number of embryogenic calli to the total number of explant used. Three independent experiments with three replicates each were carried out for each cultivar, i.e., SM, WG, and HT.

Values followed by the same superscript letters are not significantly different at $P=0.05$ according to the ANOVA test.

Table 3 Callus fresh weights in MS- based medium with different combinations and concentrations of growth hormones. 


\begin{tabular}{|c|c|c|c|c|}
\hline \multirow[t]{2}{*}{ Media } & \multirow[t]{2}{*}{$\mathrm{D}_{\mathrm{ci}}$} & \multicolumn{3}{|c|}{ Callus fresh weight (g) } \\
\hline & & SM & WG & $\mathrm{HT}$ \\
\hline CIMM1 & 11 & $1.59 \pm 0.00050^{\mathrm{a}}$ & $0.7806 \pm 0.0010^{b c}$ & $0.796 \pm 0.0045^{b c}$ \\
\hline CIMM2 & 11 & $0.897 \pm 0.0010^{a}$ & $1.321 \pm 0.0110^{b}$ & $1.355 \pm 0.0057^{c}$ \\
\hline CIMM3 & 9 & $1.619 \pm 0.0005^{a}$ & $1.043 \pm 0.0020^{\mathrm{bc}}$ & $1.114 \pm 0.0005^{\mathrm{bc}}$ \\
\hline CIMM4 & 7 & $1.496 \pm 0.0005^{a}$ & $1.112 \pm 0.0010^{b}$ & $0.519 \pm 0.0005^{c}$ \\
\hline CIMM5 & 8 & $1.318 \pm 0.0115^{a b c}$ & $1.439 \pm 0.0010^{\mathrm{ab}}$ & $1.342 \pm 0.003^{\mathrm{ac}}$ \\
\hline CIMM6 & 7 & $1.66 \pm 0.01150^{\mathrm{a}}$ & $0.104 \pm 0.0010^{\mathrm{bc}}$ & $1.31 \pm 0.0015^{\mathrm{bc}}$ \\
\hline CIMM7 & 5 & $1.816 \pm 0.0060^{\mathrm{abc}}$ & $1.772 \pm 0.0090^{\mathrm{abc}}$ & $1.754 \pm 0.0065^{a b c}$ \\
\hline CIMM8 & 8 & $1.711 \pm 0.0100^{\mathrm{a}}$ & $1.1806 \pm 0.0010^{b}$ & $1.296 \pm 0.0121^{c}$ \\
\hline
\end{tabular}

$D_{c i}$ : Days to callus initiation

Values (mean $\pm \mathrm{SE}$ ) were calculated for independent experiments with three replicates each were carried out for each cultivar, i.e., SM, WG, and HT.

Values followed by the same superscript letters are not significantly different at $P=0.05$ according to the ANOVA test.

Table 4 Responses of in vitro regeneration from embryogenic calli on MS-based medium supplemented with different growth hormones.

\begin{tabular}{|c|c|c|c|c|}
\hline \multirow[t]{2}{*}{ Media } & \multirow[t]{2}{*}{$D_{s i}$} & \multicolumn{3}{|c|}{ Regeneration frequency (\%) } \\
\hline & & SM & WG & $\mathrm{HT}$ \\
\hline RGM1 & 15 & $50.37 \pm 0.641^{\mathrm{abc}}$ & $48.29 \pm 0.513^{\mathrm{abc}}$ & $50.74 \pm 0.641^{\mathrm{abc}}$ \\
\hline RGM2 & 13 & $52.02 \pm 0.048^{\mathrm{abc}}$ & $52.69 \pm 0.801^{\mathrm{abc}}$ & $51.36 \pm 0.619^{a b c}$ \\
\hline RGM3 & 10 & $54.51 \pm 0.898^{\mathrm{abc}}$ & $55.03 \pm 0.898^{a b c}$ & $53.77 \pm 0.384^{\mathrm{abc}}$ \\
\hline RGM4 & 8 & $56.08 \pm 0.144^{\mathrm{a}}$ & $52.02 \pm 0.048^{b}$ & $62.33 \pm 0.288^{c}$ \\
\hline RGM5 & 8 & $60.74 \pm 0.74^{a}$ & $57.18 \pm 0.528^{b}$ & $62.0 \pm 0.128^{c}$ \\
\hline RGM6 & 7 & $93.83 \pm 0.384^{\mathrm{abc}}$ & $92.44 \pm 0.128^{a b c}$ & $90.23 \pm 0.387^{a b c}$ \\
\hline RGM7 & 8 & $55.03 \pm 0.777^{\mathrm{ab}}$ & $56.67 \pm 0.962^{\mathrm{ab}}$ & $62.14 \pm 0.128^{c}$ \\
\hline RGM8 & 8 & $69.68 \pm 0.721^{\mathrm{ac}}$ & $62.24 \pm 0.250^{\mathrm{b}}$ & $68.25 \pm 0.433^{\mathrm{ac}}$ \\
\hline
\end{tabular}

$D_{\text {si }}$ : Days to shoot initiation

Farequency (mean $\pm S E$ ) was calculated as the ratio of the number of regenerated shoots to the total number of calli used. Three independent experiments with three replicates each were carried out for each cultivar, i.e., SM, WG, and HT.

Values followed by the same superscript letters are not significantly different at $P=0.05$ according to the ANOVA test.

Table 5 Comparative morpho-agronomic traits performances within three indica rice genotypes, i.e., SM, WG, and HT, respectively. 


\begin{tabular}{|c|c|c|c|c|c|c|c|c|}
\hline Genotypes & $\begin{array}{l}\text { Stem length } \\
\text { (cm) }\end{array}$ & $\begin{array}{l}\text { Panicle length } \\
\text { (cm) }\end{array}$ & $\begin{array}{l}\text { Plant height } \\
\text { (cm) }\end{array}$ & $\begin{array}{l}\text { Flag leaf } \\
\text { length }(\mathrm{cm})\end{array}$ & $\begin{array}{l}\text { Flag leaf } \\
\text { width (cm) }\end{array}$ & $\begin{array}{l}\text { No. of Panicle } \\
\text { branching }\end{array}$ & No. of tiller & $\begin{array}{l}\text { No. of } \\
\text { productiv } \\
\text { tiller }\end{array}$ \\
\hline \multicolumn{9}{|l|}{ SM } \\
\hline \multirow[t]{2}{*}{ WT } & $87.25 \pm$ & $24.06 \pm$ & $111.31 \pm 0.641^{a b c}$ & $39.42 \pm$ & $1.39 \pm 0.066^{a b}$ & $17.16 \pm$ & $11.92 \pm$ & $9.33 \pm$ \\
\hline & $0.211^{\mathrm{abc}}$ & $0.644^{\mathrm{abc}}$ & & $0.342^{\mathrm{a}}$ & & $0.125^{\mathrm{ab}}$ & $0.578^{a}$ & $0.578^{a}$ \\
\hline \multirow[t]{2}{*}{ Transgenic } & $87.73 \pm$ & $24.51 \pm$ & $112.24 \pm 0.433^{a b c}$ & $39.28 \pm$ & $1.4 \pm 0.006^{\mathrm{ab}}$ & $17.83 \pm$ & $11.66 \pm$ & $9.33 \pm$ \\
\hline & $0.032^{\mathrm{abc}}$ & $0.446^{\mathrm{abc}}$ & & $0.254^{\mathrm{a}}$ & & $0.289^{a b}$ & $0.578^{a}$ & $0.578^{a}$ \\
\hline
\end{tabular}

WG

\begin{tabular}{|c|c|c|c|c|c|c|c|c|}
\hline \multirow[t]{2}{*}{ WT } & $85.95 \pm$ & $25.06 \pm$ & $111.1 \pm$ & $36.81 \pm$ & $1.49 \pm$ & $17.12 \pm$ & $9 \pm$ & $8 \pm$ \\
\hline & $0.30^{a b c}$ & $0.075^{a b c}$ & $0.389^{a b c}$ & $0.154^{\mathrm{bc}}$ & $0.3^{a b}$ & $0.267^{a b}$ & $0.82^{b c}$ & $0.438^{b c}$ \\
\hline \multirow[t]{2}{*}{ Transgenic } & $86.25 \pm 0.289^{a b c}$ & $25.06 \pm 0.058^{\mathrm{abc}}$ & $111.32 \pm 0.650^{\mathrm{abc}}$ & $37.42 \pm 0.213^{b c}$ & $1.39 \pm$ & $17.06 \pm 0.578^{\mathrm{ab}}$ & $10.66 \pm 0.578^{b c}$ & $8.33 \pm 0.5$ \\
\hline & & & & & $0.1^{\mathrm{ab}}$ & & & \\
\hline
\end{tabular}

HT

\begin{tabular}{|c|c|c|c|c|c|c|c|c|}
\hline \multirow[t]{2}{*}{ WT } & $86.97 \pm$ & $25.51 \pm$ & $112.41 \pm 0.456^{\mathrm{abc}}$ & $36.21 \pm$ & $1.28 \pm$ & $15.673 \pm 0.625^{c}$ & $10 \pm$ & $8.378 \pm 0 .\{$ \\
\hline & $0.089^{a b c}$ & $0.298^{a b c}$ & & $0.308^{b c}$ & $0.019^{c}$ & & $0.017^{b c}$ & \\
\hline \multirow[t]{2}{*}{ Transgenic } & $86.05 \pm$ & $25.32 \pm$ & $111.38 \pm 0.115^{a b c}$ & $37.62 \pm$ & $1.29 \pm$ & $15.33 \pm$ & $10.33 \pm$ & $8.33 \pm$ \\
\hline & $0.058^{\mathrm{abc}}$ & $0.058^{a b c}$ & & $0.509^{b c}$ & $0.066^{c}$ & $0.578^{c}$ & $0.578^{b c}$ & $0.578^{b c}$ \\
\hline
\end{tabular}

Values (mean \pm SE) were calculated for independent experiments with three replicates, each were carried out for each cultivar, i.e., SM, WG, and HT.

Values followed by the same superscript letters are not significantly different at $P=0.05$ according to the ANOVA test.

\section{Figures}

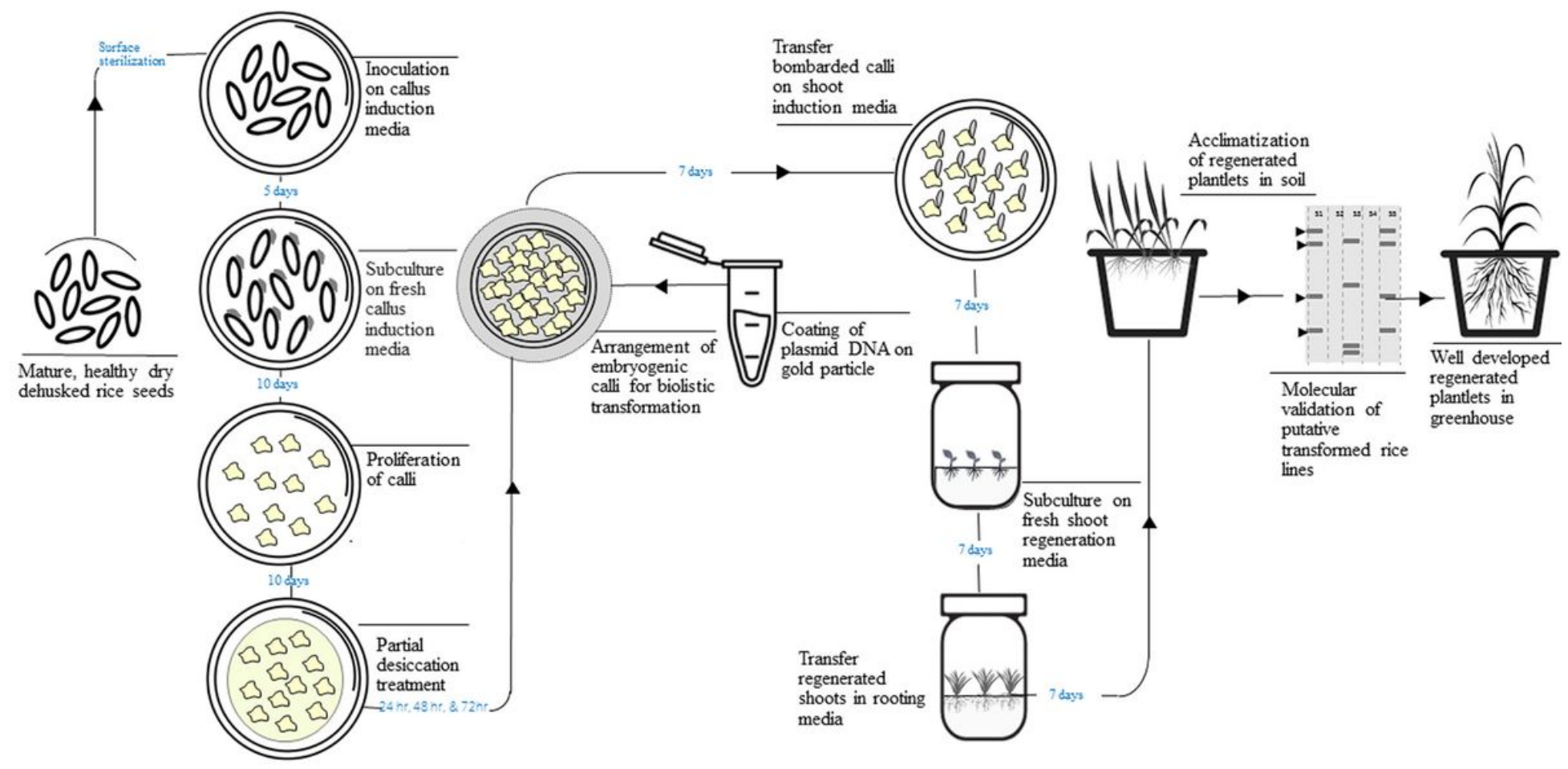

Figure 1 
Schematic representation of the standardized in vitro regeneration and transformation protocol for genotype-free indica rice cultivars. This genotype-free, simple, consistent, efficacious method may pave the way for genetic enhancement of rice genotypes employing the CRISPR/Cas-based genome editing.
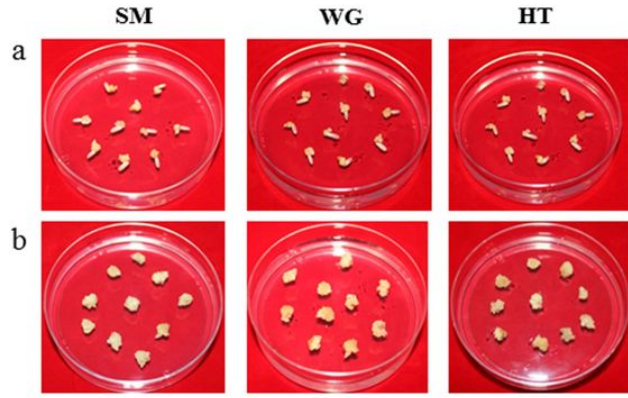
of callus
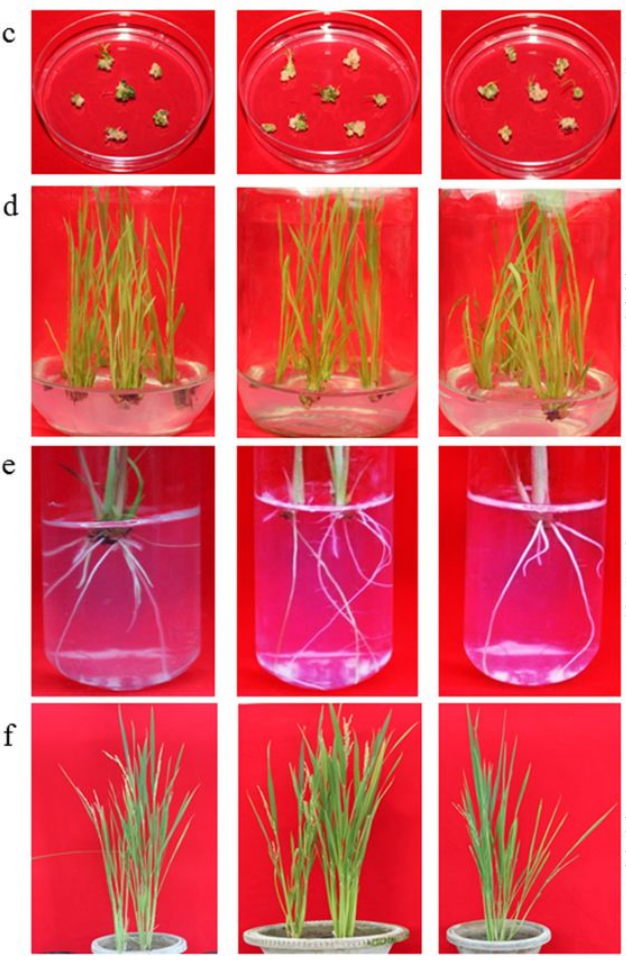

Developed Multiple shoots

Proliferation of callus

Figure 2

In vitro regeneration of three indica rice genotypes, i.e., SM, WG and HT, shows the protocol's essential stages. (a). Embryogenic calli formation in MS- and N6based callus induction media supplemented with 2,4-D ( $2.5 \mathrm{mg} / \mathrm{L})$; dicamba (1.5 mg/L); TDZ (0.1 mg/L); proline (1000 mg/L), and glutamine (2.5 mg/L) after 5 days of inoculation; (b). Proliferation of calli on respective callus induction media with similar hormonal combination; (c). Regeneration of multiple shoots from embryonic calli on MS media supplementation with BAP (1.5 mg/L); NAA (0.5 mg/L); TDZ (1.0 mg/L); zeatin ( $0.2 \mathrm{mg} / \mathrm{L})$ and proline (500 mg/L). (d). Elongation of shoots on respective regeneration media with the similar hormonal combination. Regenerated shoots were sub-cultured at 7 days interval. (e). Response of regenerated shoots to root on hormone free half-strength of MS media. (f). Well-developed regenerated plantlets showing normal growth in greenhouse. Steps $(a-b)$ had performed under dark conditions and steps (c-e) had performed under light conditions in the growth chamber under controlled temperature $\left(\mathrm{Tm}=27 \pm 1^{\circ} \mathrm{C}\right)$ \& humidity $(\mathrm{RH}=50-60 \%)$. Steps $\mathrm{f}$ had performed in greenhouse under controlled temperature \& humidity conditions $\left(\mathrm{Tm}=28 \pm 2^{\circ} \mathrm{C}\right.$; $\mathrm{RH}=85 \%)$. 


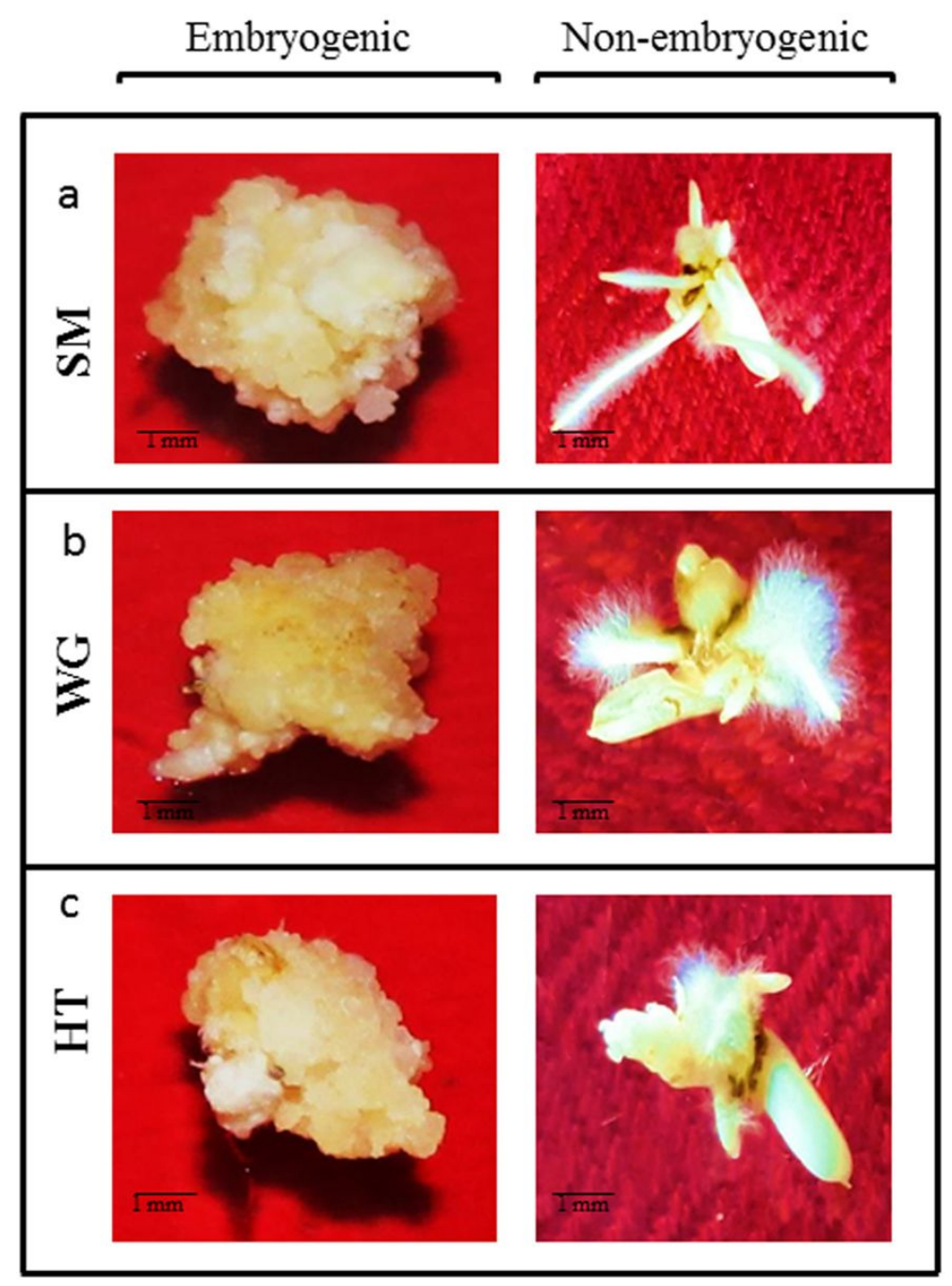

Figure 3

Morphological differences between embryogenic and non-embryogenic calli of three indica rice genotypes, i.e., SM, WT and HT. The left panel shows compact, nodular, light yellow or off-white coloured embryogenic calli, whereas the right panel shows pointed, rough, rhizogenic non-embryogenic calli.

a

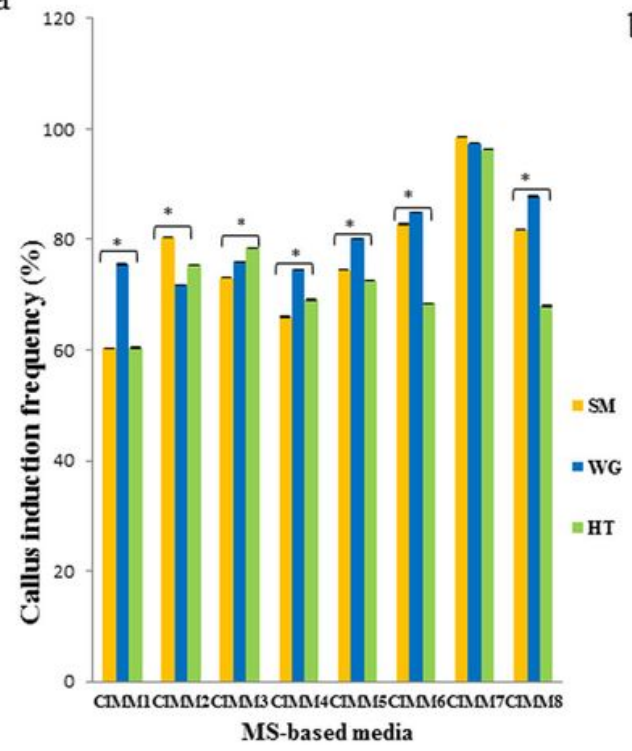

b

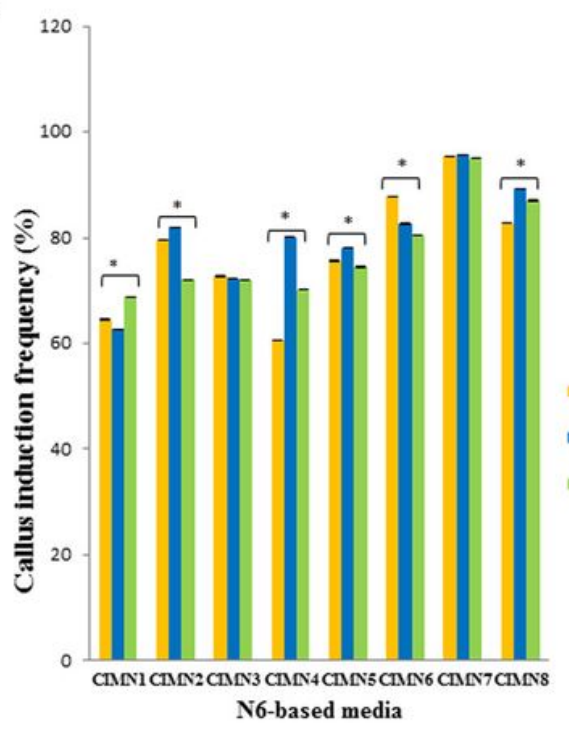

c

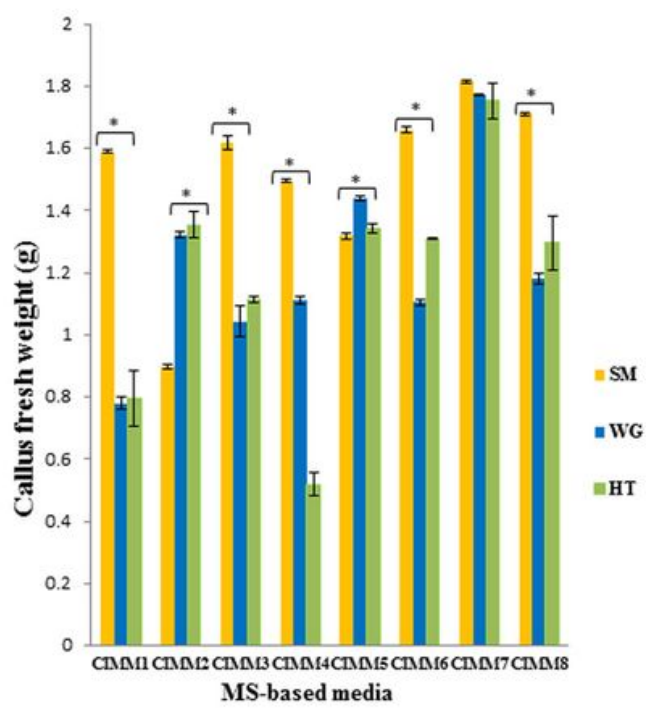

Figure 4

Callus induction frequency (\%) and fresh callus weight of three indica rice genotypes. ( $a-b)$. Responses of callus initiation on MS- and N6- based media supplemented with different growth regulators. Callus induction frequency was evaluated after two weeks of culture. Efficient CIF were revealed in all three 
genotypes on MS-based CIMM7 (96-98\%) and N6-based CIMN7 (95\%) media when supplementation with 2, 4-D (2.5 mg/L); dicamba (1.5 mg/L); TDZ (0.1 $\mathrm{mg} / \mathrm{L})$; proline (1000 mg/L); and glutamine (2.5 mg/L). (c). Fresh weight of calli for all three genotypes were recorded after $25-\mathrm{d}$ of inoculation. Calli generated on both CIMM7 \& CIMN7 media showed proliferation with increased fresh weight. CIF was recorded by the formula: CIF (\%) = No. of explants produced calli / No. of cultured explants $\times 100$. Values (mean \pm SE) were calculated for independent experiments with three replicates. For each cultivar, the statistical significance was tested using one way ANOVA $(P \leqslant 0.05)$ followed by Tukey HSD test $(H S D 0.5)$. * indicates that values within three rice genotypes are significantly different at $\mathrm{P} \leqslant 0.05$ according to the ANOVA test.

a

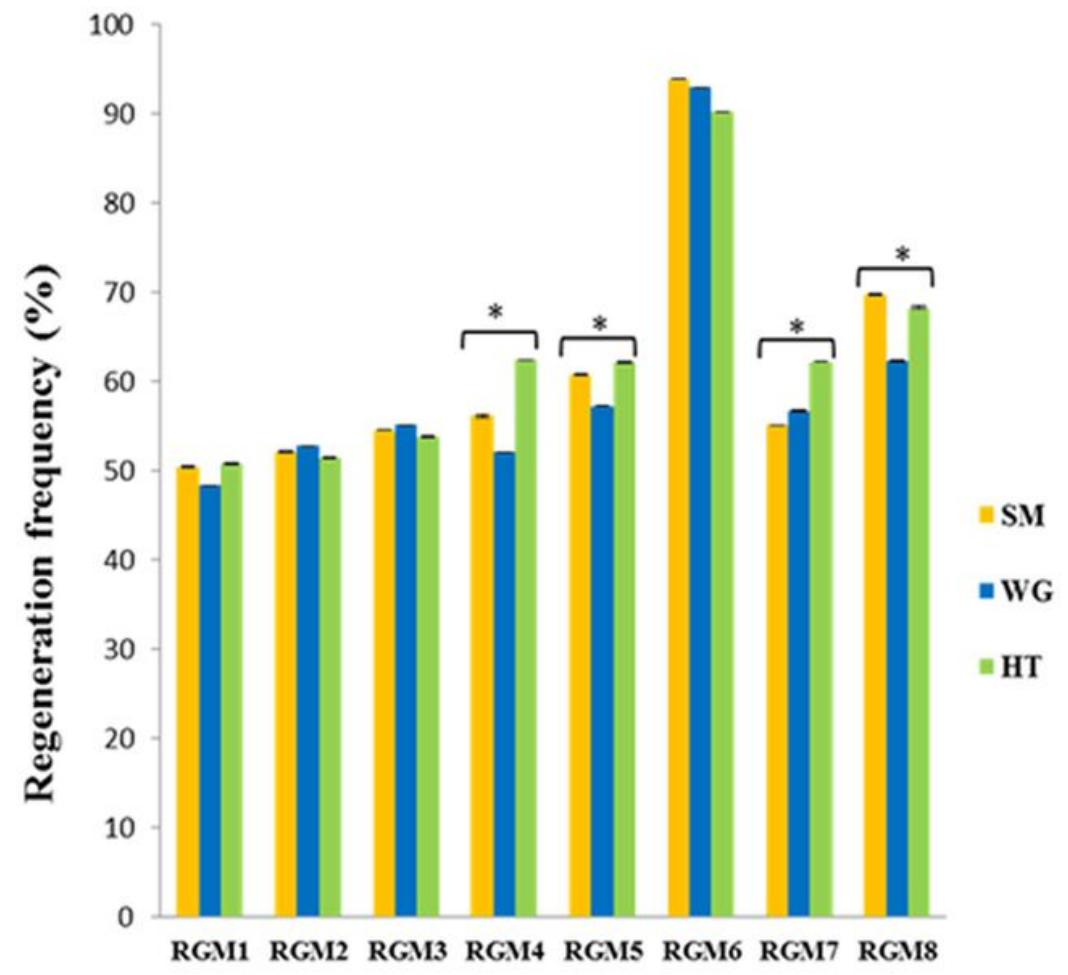

b

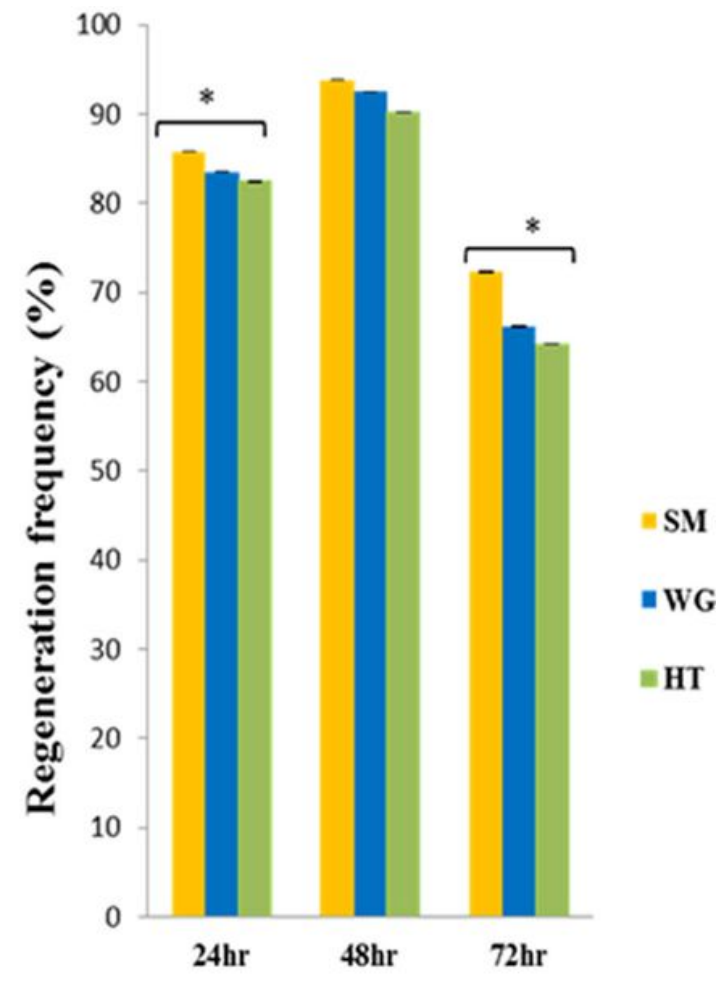

Dessiccation Duration

\section{Figure 5}

Effects of different growth regulators and partial desiccation treatment during in vitro regeneration. (a). Regeneration frequency (\%) of three indica rice cultivars, i.e., SM, WG, and HT on MS-based medium supplemented with different permutations and combinations of growth hormones. A combination of BAP $(1.5 \mathrm{mg} / \mathrm{L})$; NAA $(0.5 \mathrm{mg} / \mathrm{L})$; TDZ $(1.0 \mathrm{mg} / \mathrm{L})$; zeatin $(0.2 \mathrm{mg} / \mathrm{L})$ and proline $(500 \mathrm{mg} / \mathrm{L})$ ascertained as RGM6, revealed better regeneration performances $(90$ $94 \%)$. (b). Effect of partial desiccation was evaluated with different time point, i.,e., $24 \mathrm{hr}, 48 \mathrm{hr}$, and $72 \mathrm{hr}$, respectively. Wherein, $48 \mathrm{hr}$ desiccation treatment exhibited better regeneration performances. Regeneration frequency (RF) was recorded by the following formula: RF (\%) = No. of plants regenerated / No. of calli inoculated $\times 100$. Values (mean \pm SE) were calculated for independent experiments with three replicates. For each cultivar, the statistical significance was tested using one way ANOVA ( $\mathrm{P} \leqslant 0.05)$ followed by Tukey HSD test (HSD 0.5). * indicates that values within three rice genotypes are significantly different at $P \leqslant 0.05$ according to the ANOVA test. 


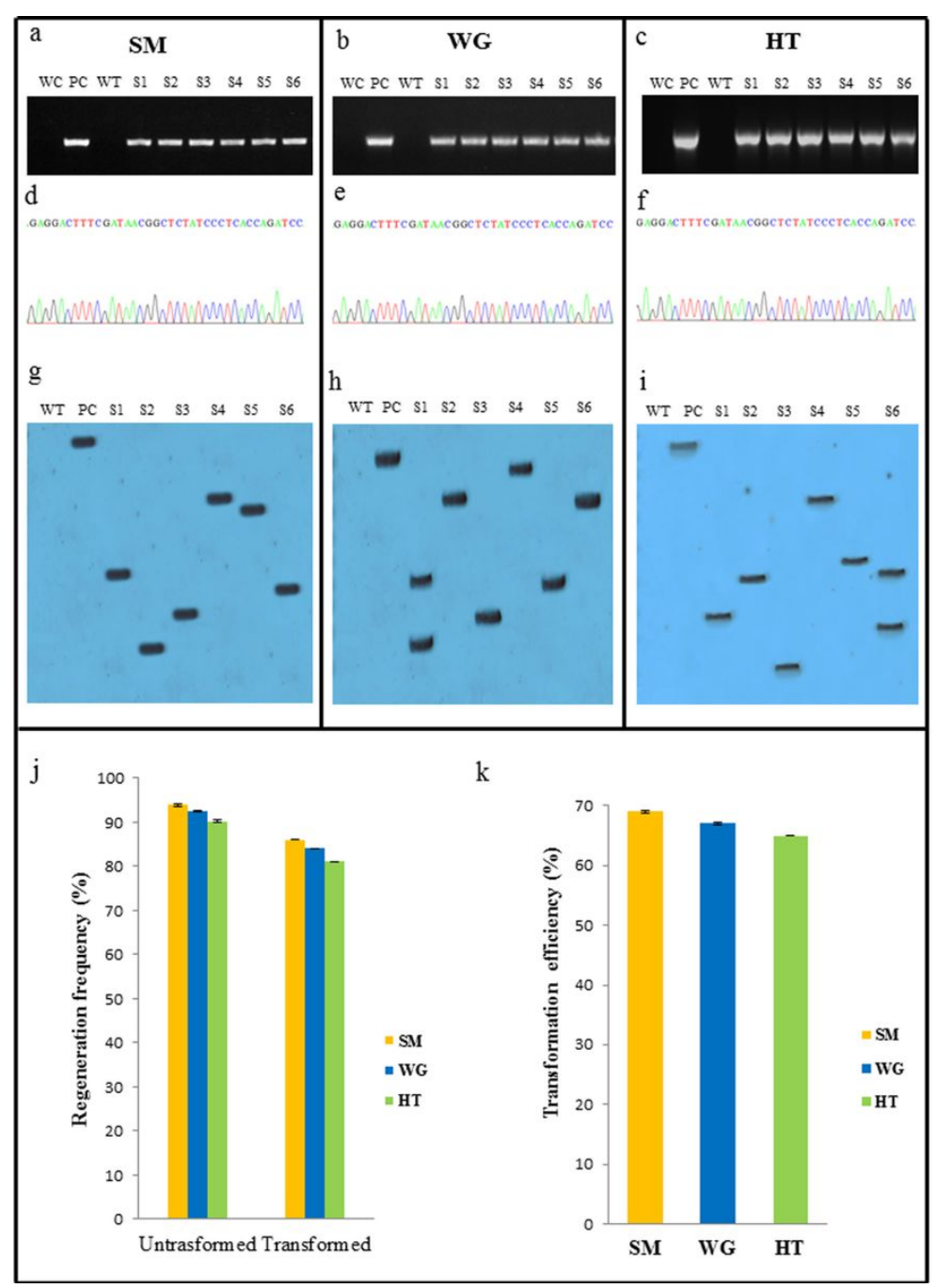

\section{Figure 6}

Confirmation of CRISPR-Cas9 reagent (Cas9gene) integration in three indica rice genotypes (SM, WG, and HT) employing biolistic transformation approach. (a-c). PCR amplification of exogenous Cas 9 gene harbouring transgenic rice lines. PCR analyses were performed using Cas 9 gene specific forward and reverse primers. The amplified lanes S1-S6 denote transgenic rice samples, where, WC- Water control, WT-wild type, and PC-Positive control (Cas9 gene). (d-f). Validation of transgenic rice lines via Sanger sequencing. ( $\mathrm{g}-\mathrm{i})$. Southern blot analysis of three transgenic rice genotypes. For southern analysis rice gDNA were digested with restriction endonuclease EcoRv and detected with DIG-labeled Cas9 probe. Southern blot signals (S1-S6) confirmed the presence and copy number of the CRISPR-Cas9 reagent (Cas9 gene) and independence of the events. Noted that no signals were detected in WT plants. Where, PC- positive control (Cas9), WT- wild type plant. (J). Regeneration frequency (\%)p of untransformed and transformed calli of three indica rice genotypes. (K).

Transformation efficiency (\%)q of three indica genotypes (SM=69\%; WG $67 \%$ and $H T=65 \%)$ using biolistic approach. pRegeneration Frequency (\%) $=$ No. of plants regenerated / No. of calli inoculated $\times 100$. qTransformation Efficiency $(\%)=$ No. of putative positive plants / No. of calli used for biolistic transformation $\times 100$. Values (mean $\pm S E$ ) were calculated for independent experiments with three replicates. For each cultivar, the statistical significance was tested using one way ANOVA $(p \leqslant 0.05)$ followed by Tukey HSD test (HSD0.5). 


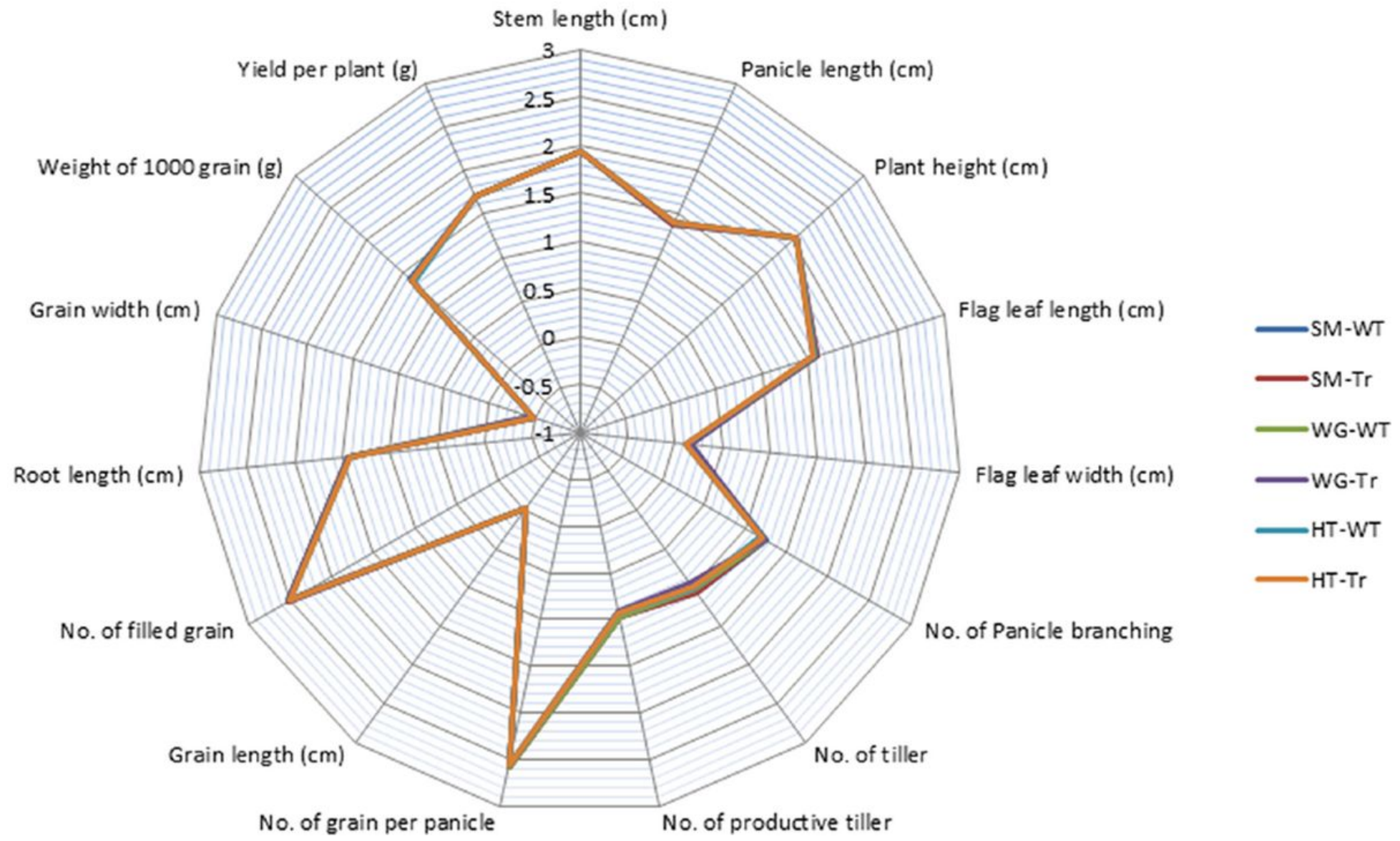

Figure 7

Comparative morpho-agronomic traits performances analysis of WT and CRISPR-Cas9 reagent (Cas9 gene) harbouring transgenic three indica rice cultivars, i.e., SM, WG, and HT, respectively. Data represent the mean \pm SE of three independent experiments $(n=3)$. The statistical significance level was performed using one-way ANOVA $(p \leqslant 0.05)$ followed by Tukey HSD test (HSD0.5).

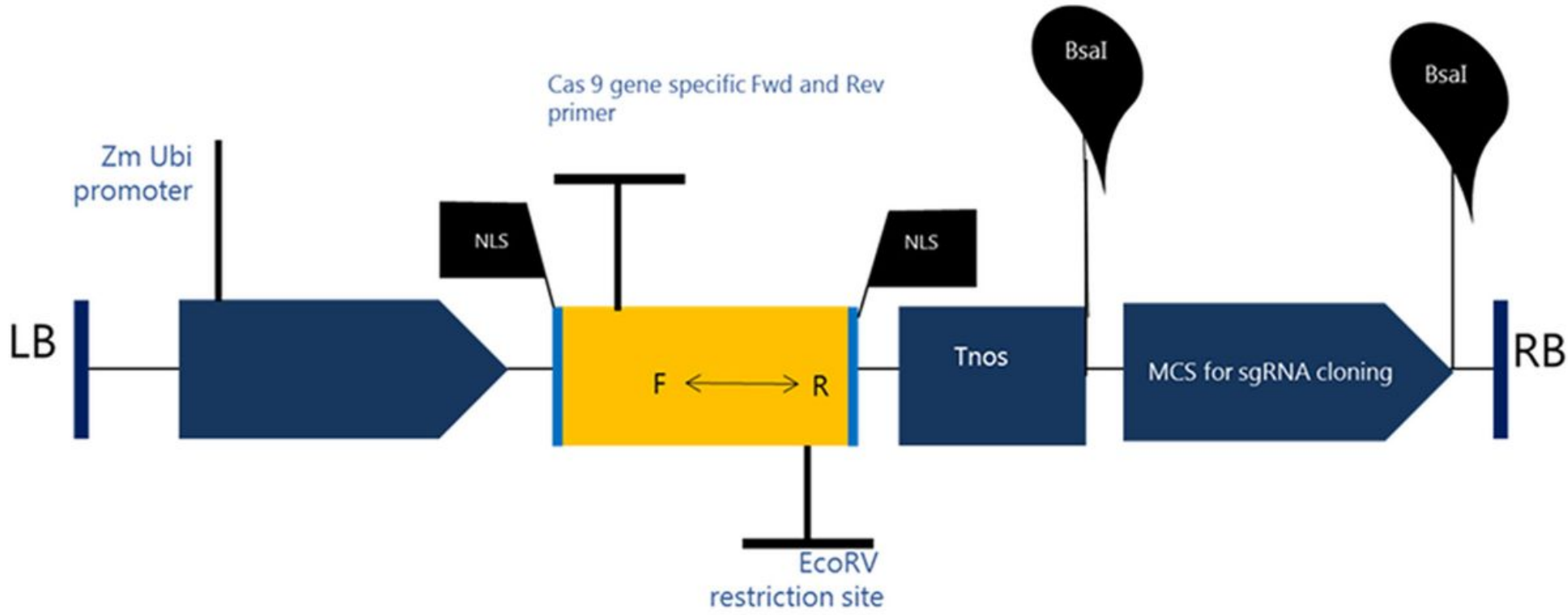

Figure 8

Schematic representation of T-DNA region of indigenously marker-free pCAMBIA1300-based plant expression vector NICTK-1_pCRISPR-Cas9 construct harbouring plant codon-optimized SpCas9 utilized for biolistic transformation of rice. The maize pUbi promoter drives the transcription of the plant codonoptimized transgene (Cas9) region that contains two SV40 NLSs at the N and C termini. Nopaline synthase (NOS) acts as a gene terminator. Bsal flanking site present at both 5' and 3' of gRNA scaffold. Multiple cloning sites (MCS) can be utilized for the integration of single or assembly of multiplexed sgRNAs for introduction of one or more agronomically important traits in plants . LB: Left border, RB: Right border. 


\section{Supplementary Files}

This is a list of supplementary files associated with this preprint. Click to download.

- AdditionalFile1Tables1.xlsx

- AdditionalFile1TableS10.xlsx

- AdditionalFile1TableS11.xlsx

- AdditionalFile1Tables12.xIsx

- AdditionalFile1Tables2.xlsx

- AdditionalFile1Tables3.xlsx

- AdditionalFile1TableS4.xIsx

- AdditionalFile1Tables5.xlsx

- AdditionalFile1Tables6.xlsx

- AdditionalFile1Tables7.xlsx

- AdditionalFile1Tables8.xIsx

- AdditionalFile1Tables9.xlsx

- Additionalfile2Fig.S1.tif

- Additionalfile2Fig.S2.tif

- Additionalfile2Fig.S3.tif

- Additionalfile2Fig.S4.tif

- Additionalfile2Fig.S5.tif

- Additionalfile2Fig.S6.tif

- Additionalfile3Fig.S1.tif

- Additionalfile3Fig.S2.tif

- Additionalfile3Fig.S3.tif

- Additionalfile3Fig.S4.tif

- Additionalfile3Fig.S5.tif

- Additionalfile3Fig.S6.tif 